\title{
A CLASS OF SECOND ORDER DIFFERENCE APPROXIMATIONS FOR SOLVING SPACE FRACTIONAL DIFFUSION EQUATIONS
}

\author{
WENYI TIAN, HAN ZHOU, AND WEIHUA DENG
}

\begin{abstract}
A class of second order approximations, called the weighted and shifted Grünwald difference (WSGD) operators, are proposed for RiemannLiouville fractional derivatives, with their effective applications to numerically solving space fractional diffusion equations in one and two dimensions. The stability and convergence of our difference schemes for space fractional diffusion equations with constant coefficients in one and two dimensions are theoretically established. Several numerical examples are implemented to test the efficiency of the numerical schemes and confirm the convergence order, and the numerical results for variable coefficients problem are also presented.
\end{abstract}

\section{INTRODUCTION}

Fractional calculus is a fundamental mathematical tool for describing some special phenomenons arising from engineering and science [15, 19, 23]. One of its most important applications is to describe the subdiffusion and superdiffusion process [5, 10, 16]. The suitable mathematical models are the diffusion equations with time and/or space fractional derivatives, where the classical first order derivative in time is replaced by the Caputo fractional derivative of order $\alpha \in(0,1)$, and the second order derivative in space is essentially replaced by the Riemann-Liouville fractional derivative of order $\alpha \in(1,2]$. The physical interpretation and practical applications of fractional diffusion equations have been discussed often with some common ideas [1,9, 14. Based on these, our main purpose of this paper is to study the higher accurate numerical solution of the space fractional diffusion equation by a novel finite difference approximation.

From the perspective of the numerical analysis, there are some fundamental difficulties in numerically approximating the fractional derivatives, because some good properties of classical approximating operators are lost. Over the last decades, the finite difference method has some developments in solving the fractional partial differential equations, e.g., [2,12, 13, 28. The Riemann-Liouville fractional derivative can be discretized by the standard Grünwald-Letnikov formula [19] with only first order accuracy, but the difference scheme based on the Grünwald-Letnikov formula for time dependent problems is unstable [12]. To overcome this problem, Meerschaert and Tadjeran in 12 first proposed the shifted Grünwald-Letnikov formula to approximate fractional advection-dispersion flow equations. Recently, second

Received by the editor January 28, 2012 and, in revised form, March 7, 2012, February 5, 2013 and November 14, 2013.

2010 Mathematics Subject Classification. Primary 26A33, 65L12, 65L20.

Key words and phrases. Riemann-Liouville fractional derivative, Fractional diffusion equation, Weighted and shifted Grünwald difference (WSGD) operator. 
order approximations to fractional derivatives have been studied, Sousaa and $\mathrm{Li}$ presented a second order discretization for the Riemann-Liouville fractional derivative and established an unconditionally stable weighted average finite difference method for the one-dimensional fractional diffusion equation in 24, and the results in two-dimensional two-sided space fractional convection diffusion equation in finite domain can be seen in 6]; Ortigueira [17] gave the "fractional centerd derivative" to approximate the Riesz fractional derivative with second order accuracy, and this method was used by Çelik and Duman in 2] to approximate the fractional diffusion equation with the Riesz fractional derivative in a finite domain. In this paper, we propose a new approach to approximate the Riemann-Liouville fractional derivative via combining the distinct shifted Grünwald-Letnikov formulae with their corresponding weights, which looks more general and flexible, and the weighted and shifted Grünwald-Letnikov formulae achieve second and higher order accuracy. A detailed algorithm shows that the weights are related to not only the shifted numbers but also the order of the fractional derivative, which implies the numerical algorithm is more related to the equation itself.

The paper is briefly summarized as follows. In Section 2 we propose a class of discrete operators to approximate the Riemann-Liouville fractional derivatives with high order truncating errors. In Sections 3 and 4 one-dimensional and twodimensional fractional diffusion equations are numerically solved by using the finite difference method based on the weighted and shifted Grünwald-Letnikov formulae, and the stability analysis of each case is presented. We prove that the finite difference solutions approximate the exact ones with $O\left(\tau^{2}+h^{2}\right)$ in the discrete $L^{2}$ norm. Some numerical experiments are performed in Section 5 to verify the efficiency and accuracy of the methods. The concluding remarks are given in Section 6 .

\section{High order approximations for Riemann-Liouville FRACTIONAL DERIVATIVES}

We begin with the definitions of the Riemann-Liouville fractional derivatives and the properties of their Fourier transform.

Definition $2.1([19])$. The $\alpha(n-1<\alpha<n)$ order left and right Riemann-Liouville fractional derivatives of the function $u(x)$ on $[a, b]$ are defined as

(1) left Riemann-Liouville fractional derivative:

$$
{ }_{a} D_{x}^{\alpha} u(x)=\frac{1}{\Gamma(n-\alpha)} \frac{\mathrm{d}^{n}}{\mathrm{~d} x^{n}} \int_{a}^{x} \frac{u(\xi)}{(x-\xi)^{\alpha-n+1}} \mathrm{~d} \xi ;
$$

(2) right Riemann-Liouville fractional derivative:

$$
{ }_{x} D_{b}^{\alpha} u(x)=\frac{(-1)^{n}}{\Gamma(n-\alpha)} \frac{\mathrm{d}^{n}}{\mathrm{~d} x^{n}} \int_{x}^{b} \frac{u(\xi)}{(\xi-x)^{\alpha-n+1}} \mathrm{~d} \xi .
$$

If $\alpha=n$, then ${ }_{a} D_{x}^{\alpha} u(x)=\frac{\mathrm{d}^{n}}{\mathrm{~d} x^{n}} u(x)$ and ${ }_{x} D_{b}^{\alpha} u(x)=(-1)^{n} \frac{\mathrm{d}^{n}}{\mathrm{~d} x^{n}} u(x)$.

Property $2.2([8])$. Let $\alpha>0, u \in C_{0}^{\infty}(\Omega), \Omega \subset \mathbb{R}$. The Fourier transforms of the left and right Riemann-Liouville fractional derivatives satisfy

$$
\begin{aligned}
& \mathscr{F}\left({ }_{-\infty} D_{x}^{\alpha} u(x)\right)=(i \omega)^{\alpha} \hat{u}(\omega), \\
& \mathscr{F}\left({ }_{x} D_{\infty}^{\alpha} u(x)\right)=(-i \omega)^{\alpha} \hat{u}(\omega),
\end{aligned}
$$


where $\hat{u}(\omega)$ denotes the Fourier transform of $u$,

$$
\hat{u}(\omega)=\int_{\mathbb{R}} \mathrm{e}^{-i \omega x} u(x) \mathrm{d} x .
$$

In [12, the shifted Grünwald difference operator

$$
A_{h, p}^{\alpha} u(x)=\frac{1}{h^{\alpha}} \sum_{k=0}^{\infty} g_{k}^{(\alpha)} u(x-(k-p) h),
$$

approximates the left Riemann-Liouville fractional derivative uniformly with first order accuracy, i.e.,

$$
A_{h, p}^{\alpha} u(x)={ }_{-\infty} D_{x}^{\alpha} u(x)+O(h),
$$

where $p$ is an integer and $g_{k}^{(\alpha)}=(-1)^{k}\left(\begin{array}{l}\alpha \\ k\end{array}\right)$. In fact, the coefficients $g_{k}^{(\alpha)}$ in (2.1) are the coefficients of the power series of the function $(1-z)^{\alpha}$,

$$
(1-z)^{\alpha}=\sum_{k=0}^{\infty}(-1)^{k}\left(\begin{array}{l}
\alpha \\
k
\end{array}\right) z^{k}=\sum_{k=0}^{\infty} g_{k}^{(\alpha)} z^{k},
$$

for all $|z| \leq 1$, and they can be evaluated recursively:

$$
g_{0}^{(\alpha)}=1, \quad g_{k}^{(\alpha)}=\left(1-\frac{\alpha+1}{k}\right) g_{k-1}^{(\alpha)}, k=1,2, \ldots
$$

Lemma 2.3 ([12,13, 19]). The coefficients in (2.1) satisfy the following properties for $1<\alpha \leq 2$ :

$$
\left\{\begin{array}{l}
g_{0}^{(\alpha)}=1, g_{1}^{(\alpha)}=-\alpha<0 \\
1 \geq g_{2}^{(\alpha)} \geq g_{3}^{(\alpha)} \geq \ldots \geq 0 \\
\sum_{k=0}^{\infty} g_{k}^{(\alpha)}=0, \sum_{k=0}^{m} g_{k}^{(\alpha)}<0, m \geq 1 .
\end{array}\right.
$$

2.1. Second order approximations. Inspired by the shifted Grünwald difference operator (2.1) and multi-step method, we derive the following second order approximation for the Riemann-Liouville fractional derivatives.

Theorem 2.4. Let $u \in L^{1}(\mathbb{R}),{ }_{-\infty} D_{x}^{\alpha+2} u$ and its Fourier transform belong to $L^{1}(\mathbb{R})$, and define the weighted and shifted Grünwald difference (WSGD) operator by

$$
{ }_{L} \mathcal{D}_{h, p, q}^{\alpha} u(x)=\frac{\alpha-2 q}{2(p-q)} A_{h, p}^{\alpha} u(x)+\frac{2 p-\alpha}{2(p-q)} A_{h, q}^{\alpha} u(x),
$$

then we have

$$
{ }_{L} \mathcal{D}_{h, p, q}^{\alpha} u(x)={ }_{-\infty} D_{x}^{\alpha} u(x)+O\left(h^{2}\right)
$$

uniformly for $x \in \mathbb{R}$, where $p, q$ are integers and $p \neq q$.

Note. The role of $p$ and $q$ is symmetric, i.e., ${ }_{L} \mathcal{D}_{h, p, q}^{\alpha} u(x)={ }_{L} \mathcal{D}_{h, q, p}^{\alpha} u(x)$.

Proof. By the definition of $A_{h, p}^{\alpha}$ in (2.1), we assume the WSGD operator with the following form:

$$
{ }_{L} \mathcal{D}_{h, p, q}^{\alpha} u(x)=\frac{\lambda_{1}}{h^{\alpha}} \sum_{k=0}^{\infty} g_{k}^{(\alpha)} u(x-(k-p) h)+\frac{\lambda_{2}}{h^{\alpha}} \sum_{k=0}^{\infty} g_{k}^{(\alpha)} u(x-(k-q) h) .
$$


Taking Fourier transform on (2.8), we obtain

$$
\begin{aligned}
\mathscr{F}\left[{ }_{L} \mathcal{D}_{h, p, q}^{\alpha} u\right](\omega) & =\frac{1}{h^{\alpha}} \sum_{k=0}^{\infty} g_{k}^{(\alpha)}\left(\lambda_{1} \mathrm{e}^{-i \omega(k-p) h}+\lambda_{2} \mathrm{e}^{-i \omega(k-q) h}\right) \hat{u}(\omega) \\
& =\frac{1}{h^{\alpha}}\left(\lambda_{1}\left(1-\mathrm{e}^{-i \omega h}\right)^{\alpha} \mathrm{e}^{i \omega h p}+\lambda_{2}\left(1-\mathrm{e}^{-i \omega h}\right)^{\alpha} \mathrm{e}^{i \omega h q}\right) \hat{u}(\omega) \\
& =(i \omega)^{\alpha}\left(\lambda_{1} W_{p}(i \omega h)+\lambda_{2} W_{q}(i \omega h)\right) \hat{u}(\omega),
\end{aligned}
$$

where

$$
W_{r}(z)=\left(\frac{1-\mathrm{e}^{-z}}{z}\right)^{\alpha} \mathrm{e}^{r z}=1+\left(r-\frac{\alpha}{2}\right) z+O\left(z^{2}\right), r=p, q
$$

In order to have second order accuracy, coefficients $\lambda_{1}$ and $\lambda_{2}$ satisfy

$$
\left\{\begin{array}{l}
\lambda_{1}+\lambda_{2}=1 \\
\left(p-\frac{\alpha}{2}\right) \lambda_{1}+\left(q-\frac{\alpha}{2}\right) \lambda_{2}=0
\end{array}\right.
$$

which indicates that $\lambda_{1}=\frac{\alpha-2 q}{2(p-q)}$ and $\lambda_{2}=\frac{2 p-\alpha}{2(p-q)}$.

Denoting $\hat{\phi}(\omega, h)=\mathscr{F}\left[{ }_{L} \mathcal{D}_{h, p, q}^{\alpha} u\right](\omega)-\mathscr{F}\left[{ }_{-\infty} D_{x}^{\alpha} u\right](\omega)$, then from (2.9) and (2.10) there exists

$$
|\hat{\phi}(\omega, h)| \leq C h^{2}|i \omega|^{\alpha+2}|\hat{u}(\omega)|
$$

Using the condition $\mathscr{F}\left[-\infty D_{x}^{\alpha+2} u\right](\omega) \in L^{1}(\mathbb{R})$, yields

$$
\begin{aligned}
\left|{ }_{L} \mathcal{D}_{h, p, q}^{\alpha} u-{ }_{-\infty} D_{x}^{\alpha} u\right|=|\phi| & \leq \frac{1}{2 \pi} \int_{\mathbb{R}}|\hat{\phi}(\omega, h)| \\
& \leq C\left\|\mathscr{F}\left[-_{-\infty} D_{x}^{\alpha+2} u\right](\omega)\right\|_{L^{1}} h^{2}=O\left(h^{2}\right) .
\end{aligned}
$$

Remark 2.5. For the right Riemann-Liouville fractional derivative, similar to Theorem 2.4, we can check that

$$
{ }_{R} \mathcal{D}_{h, p, q}^{\alpha} u(x)=\frac{\alpha-2 q}{2(p-q)} B_{h, p}^{\alpha} u(x)+\frac{2 p-\alpha}{2(p-q)} B_{h, q}^{\alpha} u(x)={ }_{x} D_{\infty}^{\alpha} u(x)+O\left(h^{2}\right),
$$

uniformly for $x \in \mathbb{R}$ under the conditions that $u \in L^{1}(\mathbb{R}),{ }_{x} D_{\infty}^{\alpha+2} u$ and its Fourier transform belong to $L^{1}(\mathbb{R})$, where $p, q$ are integers and

$$
B_{h, r}^{\alpha} u(x)=\frac{1}{h^{\alpha}} \sum_{k=0}^{\infty} g_{k}^{(\alpha)} u(x+(k-r) h) .
$$

Remark 2.6. Considering a well-defined function $u(x)$ on the bounded interval $[a, b]$, if $u(a)=0$ or $u(b)=0$, the function $u(x)$ can be zero extended for $x<a$ or $x>b$. Then the $\alpha$ order left and right Riemann-Liouville fractional derivatives of $u(x)$ at each point $x$ can be approximated by the WSGD operators with second order 
accuracy

$$
\begin{aligned}
{ }_{a} D_{x}^{\alpha} u(x)= & \frac{\lambda_{1}}{h^{\alpha}} \sum_{k=0}^{\left[\frac{x-a}{h}\right]+p} g_{k}^{(\alpha)} u(x-(k-p) h) \\
& +\frac{\lambda_{2}}{h^{\alpha}} \sum_{k=0}^{\left[\frac{x-a}{h}\right]+q} g_{k}^{(\alpha)} u(x-(k-q) h)+O\left(h^{2}\right), \\
{ }_{x} D_{b}^{\alpha} u(x)= & \frac{\lambda_{1}}{h^{\alpha}} \sum_{k=0}^{\left[\frac{b-x}{h}\right]+p} g_{k}^{(\alpha)} u(x+(k-p) h) \\
& +\frac{\lambda_{2}}{h^{\alpha}} \sum_{k=0}^{\left[\frac{b-x}{h}\right]+q} g_{k}^{(\alpha)} u(x+(k-q) h)+O\left(h^{2}\right),
\end{aligned}
$$

where $\lambda_{1}=\frac{\alpha-2 q}{2(p-q)}, \lambda_{2}=\frac{2 p-\alpha}{2(p-q)}$.

Remark 2.7. The integers $p, q$ are the numbers of the points located on the right/left sides of the point $x$ used for evaluating the $\alpha$ order left/right Riemann-Liouville fractional derivatives at $x$, thus, when employing the difference method with (2.15) for approximating non-periodic fractional differential equations on bounded interval, $p, q$ should be chosen satisfying $|p| \leq 1,|q| \leq 1$ to ensure that the nodes at which the values of $u$ needed in (2.15) are within the bounded interval; otherwise, we need to use another way to discretize the fractional derivative when $x$ is close to the right/left boundary. When $(p, q)=(0,-1)$, the approximation method turns out to be unstable for time dependent problems. So two sets of $(p, q)$ can be selected to establish the difference scheme for fractional diffusion equations, that is, $(1,0),(1,-1)$, and the corresponding weights in (2.6) and (2.13) are $\left(\frac{\alpha}{2}, \frac{2-\alpha}{2}\right)$ and $\left(\frac{2+\alpha}{4}, \frac{2-\alpha}{4}\right)$. For $\alpha=2$, the WSGD operator (2.6) is the centered difference approximation of second order derivative when $(p, q)$ is equal to $(1,0)$ or $(1,-1)$; for $\alpha=1,(p, q)=(1,0)$, the centered difference scheme for first order derivative is recovered.

The simplified forms of the discrete approximations (2.15) for Riemann-Liouville fractional derivatives on grid points $\left\{x_{i}=a+i h, h=(b-a) / n, i=1, \ldots, n-1\right\}$ with $(p, q)=(1,0),(1,-1)$ are

$$
\begin{aligned}
& { }_{a} D_{x}^{\alpha} u\left(x_{i}\right)=\frac{1}{h^{\alpha}} \sum_{k=0}^{i+1} w_{k}^{(\alpha)} u\left(x_{i-k+1}\right)+O\left(h^{2}\right), \\
& { }_{x} D_{b}^{\alpha} u\left(x_{i}\right)=\frac{1}{h^{\alpha}} \sum_{k=0}^{N-i+1} w_{k}^{(\alpha)} u\left(x_{i+k-1}\right)+O\left(h^{2}\right),
\end{aligned}
$$

where

$$
\left\{\begin{aligned}
&(p, q)=(1,0), w_{0}^{(\alpha)}=\frac{\alpha}{2} g_{0}^{(\alpha)}, w_{k}^{(\alpha)}=\frac{\alpha}{2} g_{k}^{(\alpha)}+\frac{2-\alpha}{2} g_{k-1}^{(\alpha)}, k \geq 1, \\
&(p, q)=(1,-1), \quad w_{0}^{(\alpha)}=\frac{2+\alpha}{4} g_{0}^{(\alpha)}, w_{1}^{(\alpha)}=\frac{2+\alpha}{4} g_{1}^{(\alpha)} \\
& w_{k}^{(\alpha)}=\frac{2+\alpha}{4} g_{k}^{(\alpha)}+\frac{2-\alpha}{4} g_{k-2}^{(\alpha)}, k \geq 2 .
\end{aligned}\right.
$$


With Lemma 2.3 and some calculations, we obtain the properties of the coefficients $w_{k}^{(\alpha)}$ in (2.16) corresponding to $(p, q)=(1,0),(1,-1)$ as follows.

Lemma 2.8. The coefficients in (2.16) satisfy the following properties for $1<\alpha \leq 2$,

(1) if $(p, q)=(1,0)$,

$$
\left\{\begin{array}{l}
w_{0}^{(\alpha)}=\frac{\alpha}{2}, w_{1}^{(\alpha)}=\frac{2-\alpha-\alpha^{2}}{2}<0, w_{2}^{(\alpha)}=\frac{\alpha\left(\alpha^{2}+\alpha-4\right)}{4}, \\
1 \geq w_{0}^{(\alpha)} \geq w_{3}^{(\alpha)} \geq w_{4}^{(\alpha)} \geq \ldots \geq 0 \\
\sum_{k=0}^{\infty} w_{k}^{(\alpha)}=0, \sum_{k=0}^{m} w_{k}^{(\alpha)}<0, m \geq 2
\end{array}\right.
$$

(2) if $(p, q)=(1,-1)$,

$$
\left\{\begin{array}{l}
w_{0}^{(\alpha)}=\frac{2+\alpha}{4}, w_{1}^{(\alpha)}=-\frac{2 \alpha+\alpha^{2}}{4}<0, \\
w_{2}^{(\alpha)}=\frac{\alpha^{3}+\alpha^{2}-4 \alpha+4}{8}>0, w_{3}^{(\alpha)}=\frac{\alpha(2-\alpha)\left(\alpha^{2}+\alpha-8\right)}{6} \leq 0, \\
1 \geq w_{0}^{(\alpha)} \geq w_{2}^{(\alpha)} \geq w_{4}^{(\alpha)} \geq w_{5}^{(\alpha)} \geq \ldots \geq 0, \\
\sum_{k=0}^{\infty} w_{k}^{(\alpha)}=0, \sum_{k=0}^{m} w_{k}^{(\alpha)}<0, m=1 \text { or } m \geq 3 .
\end{array}\right.
$$

Next, we will explore the properties of the eigenvalues of the difference matrix of (2.16) on grid points $\left\{x_{k}=a+k h, h=(b-a) / n, k=1,2, \ldots, n-1\right\}$. In the following, we denote by $H$ the symmetric (respectively, hermitian) part of $A$ if $\mathrm{A}$ is real (respectively, complex) matrix.

Lemma 2.9 ([21]). A real matrix $A$ of order $n$ is positive definite if and only if its symmetric part $H=\frac{A+A^{T}}{2}$ is positive definite; $H$ is positive definite if and only if the eigenvalues of $H$ are positive.

Lemma $2.10([21])$. If $A \in \mathbb{C}^{n \times n}$, let $H=\frac{A+A^{*}}{2}$ be the hermitian part of $A, A^{*}$ the conjugate transpose of $A$, then for any eigenvalue $\lambda$ of $A$, there exists

$$
\lambda_{\min }(H) \leq \operatorname{Re}(\lambda) \leq \lambda_{\max }(H),
$$

where $\operatorname{Re}(\lambda)$ represents the real part of $\lambda$, and $\lambda_{\min }(H), \lambda_{\max }(H)$ are the minimum and maximum of the eigenvalues of $H$.

Definition 2.11 ([4]). Let Toeplitz matrix $T_{n}$ be of the following form:

$$
T_{n}=\left(\begin{array}{ccccc}
t_{0} & t_{-1} & \cdots & t_{2-n} & t_{1-n} \\
t_{1} & t_{0} & t_{-1} & \cdots & t_{2-n} \\
\vdots & t_{1} & t_{0} & \ddots & \vdots \\
t_{n-2} & \cdots & \ddots & \ddots & t_{-1} \\
t_{n-1} & t_{n-2} & \cdots & t_{1} & t_{0}
\end{array}\right)
$$

If the diagonals $\left\{t_{k}\right\}_{k=-n+1}^{n-1}$ are the Fourier coefficients of a function $f$, i.e.,

$$
t_{k}=\frac{1}{2 \pi} \int_{-\pi}^{\pi} f(x) e^{-i k x} d x
$$

then the function $f$ is called the generating function of $T_{n}$. 
Lemma 2.12 (Grenander-Szegö theorem [3,4]). For the above Toeplitz matrix $T_{n}$, if $f$ is a $2 \pi$-periodic continuous real-valued function, denote $\lambda_{\min }\left(T_{n}\right)$ and $\lambda_{\max }\left(T_{n}\right)$ as the smallest and largest eigenvalues of $T_{n}$, respectively. Then we have

$$
f_{\min } \leq \lambda_{\min }\left(T_{n}\right) \leq \lambda_{\max }\left(T_{n}\right) \leq f_{\max },
$$

where $f_{\min }, f_{\max }$ denote the minimum and maximum values of $f(x)$. Moreover, if $f_{\min }<f_{\max }$, then all eigenvalues of $T_{n}$ satisfy

$$
f_{\min }<\lambda\left(T_{n}\right)<f_{\max },
$$

for all $n>0$; furthermore if $f_{\min } \geq 0$, then $T_{n}$ is positive definite.

Theorem 2.13. Let matrix $A$ be of the form

$$
A=\left(\begin{array}{ccccc}
w_{1}^{(\alpha)} & w_{0}^{(\alpha)} & & & \\
w_{2}^{(\alpha)} & w_{1}^{(\alpha)} & w_{0}^{(\alpha)} & & \\
\vdots & w_{2}^{(\alpha)} & w_{1}^{(\alpha)} & \ddots & \\
w_{n-2}^{(\alpha)} & \ldots & \ddots & \ddots & w_{0}^{(\alpha)} \\
w_{n-1}^{(\alpha)} & w_{n-2}^{(\alpha)} & \cdots & w_{2}^{(\alpha)} & w_{1}^{(\alpha)}
\end{array}\right)
$$

where the diagonals $\left\{w_{k}^{(\alpha)}\right\}_{k=0}^{n-1}$ are the coefficients given in (2.16) corresponding to $(p, q)=(1,0)$ or $(1,-1)$. Then we have that any eigenvalue $\lambda$ of $A$ satisfies

(1) $\operatorname{Re}(\lambda) \equiv 0$, for $(p, q)=(1,0), \alpha=1$,

(2) $\operatorname{Re}(\lambda)<0$, for $(p, q)=(1,0), 1<\alpha \leq 2$,

(3) $\operatorname{Re}(\lambda)<0$, for $(p, q)=(1,-1), 1 \leq \alpha \leq 2$.

Moreover, when $1<\alpha \leq 2$, matrix $A$ is negative definite, and the real parts of the eigenvalues of matrix $c_{1} A+c_{2} A^{\mathrm{T}}$ are less than 0 , where $c_{1}, c_{2} \geq 0, c_{1}^{2}+c_{2}^{2} \neq 0$.

Proof. We consider the symmetric part of matrix $A$, denoted as $H=\frac{A+A^{T}}{2}$. The generating functions of $A$ and $A^{T}$ are

$$
f_{A}(x)=\sum_{k=0}^{\infty} w_{k}^{(\alpha)} \mathrm{e}^{i(k-1) x}, \quad f_{A^{T}}(x)=\sum_{k=0}^{\infty} w_{k}^{(\alpha)} \mathrm{e}^{-i(k-1) x},
$$

respectively. Then $f(\alpha ; x)=\frac{f_{A}(x)+f_{A^{T}}(x)}{2}$ is the generating function of $H$, and $f(\alpha ; x)$ is a periodic continuous real-valued function on $[-\pi, \pi]$ since $f_{A}(x)$ and $f_{A^{T}}(x)$ are mutually conjugated.

Case $(p, q)=(1,0)$ : with the corresponding coefficients $w_{k}^{(\alpha)}$ given by (2.17), then

$$
\begin{aligned}
f(\alpha ; x)= & \frac{1}{2}\left(\sum_{k=0}^{\infty} w_{k}^{(\alpha)} \mathrm{e}^{i(k-1) x}+\sum_{k=0}^{\infty} w_{k}^{(\alpha)} \mathrm{e}^{-i(k-1) x}\right) \\
= & \frac{1}{2}\left(\frac{\alpha}{2} \mathrm{e}^{-i x} \sum_{k=0}^{\infty} g_{k}^{(\alpha)} \mathrm{e}^{i k x}+\frac{2-\alpha}{2} \sum_{k=0}^{\infty} g_{k}^{(\alpha)} \mathrm{e}^{i k x}\right. \\
& \left.+\frac{\alpha}{2} \mathrm{e}^{i x} \sum_{k=0}^{\infty} g_{k}^{(\alpha)} \mathrm{e}^{-i k x}+\frac{2-\alpha}{2} \sum_{k=0}^{\infty} g_{k}^{(\alpha)} \mathrm{e}^{-i k x}\right) \\
= & \frac{\alpha}{4}\left(\mathrm{e}^{-i x}\left(1-\mathrm{e}^{i x}\right)^{\alpha}+\mathrm{e}^{i x}\left(1-\mathrm{e}^{-i x}\right)^{\alpha}\right)+\frac{2-\alpha}{4}\left(\left(1-\mathrm{e}^{i x}\right)^{\alpha}+\left(1-\mathrm{e}^{-i x}\right)^{\alpha}\right) .
\end{aligned}
$$


Next we check $f(\alpha ; x) \leq 0$ for $1<\alpha \leq 2$. Since $f(\alpha ; x)$ is a real-valued and even function, we just consider its principal value on $[0, \pi]$. By the formula

$$
\mathrm{e}^{i \theta}-\mathrm{e}^{i \phi}=2 i \sin \left(\frac{\theta-\phi}{2}\right) \mathrm{e}^{\frac{i(\theta+\phi)}{2}},
$$

we obtain

$$
f(\alpha ; x)=\left(2 \sin \left(\frac{x}{2}\right)\right)^{\alpha}\left(\frac{\alpha}{2} \cos \left(\frac{\alpha}{2}(x-\pi)-x\right)+\frac{2-\alpha}{2} \cos \left(\frac{\alpha}{2}(x-\pi)\right)\right) .
$$

Denoting

$$
g(\alpha ; x)=\frac{\alpha}{2} \cos \left(\frac{\alpha}{2}(x-\pi)-x\right)+\frac{2-\alpha}{2} \cos \left(\frac{\alpha}{2}(x-\pi)\right),
$$

it is easy to prove that $g(\alpha ; x)$ decreases with respect to $\alpha$, then $f(\alpha ; x) \leq 0$. By Lemma 2.10 and 2.12, $\operatorname{Re}(\lambda) \equiv 0$ for $\alpha=1$ as $f(1 ; x) \equiv 0$, and $f(\alpha ; x)$ is not identically zero for $1<\alpha \leq 2$, then we get $\operatorname{Re}(\lambda)<0$.

Case $(p, q)=(1,-1)$ : the corresponding generating function $f(\alpha ; x)$ of $\frac{A+A^{T}}{2}$ can be calculated in the following form with coefficients $w_{k}^{(\alpha)}$ given by (2.17),

$$
\begin{aligned}
f(\alpha ; x)= & \frac{1}{2}\left(\sum_{k=0}^{\infty} w_{k}^{(\alpha)} \mathrm{e}^{i(k-1) x}+\sum_{k=0}^{\infty} w_{k}^{(\alpha)} \mathrm{e}^{-i(k-1) x}\right) \\
= & \frac{2+\alpha}{8}\left(\mathrm{e}^{-i x} \sum_{k=0}^{\infty} g_{k}^{(\alpha)} \mathrm{e}^{i k x}+\mathrm{e}^{i x} \sum_{k=0}^{\infty} g_{k}^{(\alpha)} \mathrm{e}^{-i k x}\right) \\
& +\frac{2-\alpha}{8}\left(\mathrm{e}^{i x} \sum_{k=0}^{\infty} g_{k}^{(\alpha)} \mathrm{e}^{i k x}+\mathrm{e}^{-i x} \sum_{k=0}^{\infty} g_{k}^{(\alpha)} \mathrm{e}^{-i k x}\right) \\
= & \frac{2+\alpha}{8}\left(\mathrm{e}^{-i x}\left(1-\mathrm{e}^{i x}\right)^{\alpha}+\mathrm{e}^{i x}\left(1-\mathrm{e}^{-i x}\right)^{\alpha}\right) \\
& +\frac{2-\alpha}{8}\left(\mathrm{e}^{i x}\left(1-\mathrm{e}^{i x}\right)^{\alpha}+\mathrm{e}^{-i x}\left(1-\mathrm{e}^{-i x}\right)^{\alpha}\right) .
\end{aligned}
$$

Next we check $f(\alpha ; x) \leq 0$ for $1<\alpha \leq 2$. Since $f(\alpha ; x)$ is a real-valued and even function, we just consider its principal value on $[0, \pi]$. By simple calculation, we obtain

$$
f(\alpha ; x)=\left(2 \sin \left(\frac{x}{2}\right)\right)^{\alpha}\left(\frac{\alpha}{2} \sin \left(\frac{\alpha}{2}(x-\pi)\right) \sin (x)+\cos \left(\frac{\alpha}{2}(x-\pi)\right) \cos (x)\right) .
$$

Denoting

$$
g(\alpha ; x)=\frac{\alpha}{2} \sin \left(\frac{\alpha}{2}(x-\pi)\right) \sin (x)+\cos \left(\frac{\alpha}{2}(x-\pi)\right) \cos (x),
$$

we can also check that $g(\alpha ; x)$ decreases with respect to $\alpha$, then

$$
f(\alpha ; x) \leq\left(2 \sin \left(\frac{x}{2}\right)\right)^{\alpha} g(1 ; x)=-\left(2 \sin \left(\frac{x}{2}\right)\right)^{\alpha} \sin ^{3}\left(\frac{x}{2}\right) \leq 0 .
$$

Therefore, by Lemma 2.10 and 2.12, we get $\operatorname{Re}(\lambda)<0$ for $1 \leq \alpha \leq 2$.

From the above discussions and Lemma 2.12 we know, for $1<\alpha \leq 2$, the matrix $\frac{1}{2}\left(A+A^{T}\right)$ is negative definite, which implies matrix $A$ is negative definite by Lemma 2.9, and the symmetric part of matrix $c_{1} A+c_{2} A^{\mathrm{T}}$ is $\frac{c_{1}+c_{2}}{2}\left(A+A^{\mathrm{T}}\right)$; thus we obtain $\operatorname{Re}\left(\lambda\left(c_{1} A+c_{2} A^{\mathrm{T}}\right)\right)<0$ for $1<\alpha \leq 2$. 
Remark 2.14. For the case $(p, q)=(1,0)$ and $1<\alpha \leq 2$, we can check that the symmetric part $H$ of matrix $A$ in (2.20) is strictly diagonally dominant by using Lemma 2.8, and the elements of the main diagonal of $H$ are negative, then the eigenvalues of $H$ are less than zero by the Gershgorin circle theorem ([21], p. 188), therefore, with Lemmas 2.9 and 2.10 , we can also get $\operatorname{Re}(\lambda(A))<0$, and $A$ is negative definite.

Remark 2.15. By the same approach described in Theorem 2.13, we can verify that the generating function of the symmetric part of difference matrix for $(p, q)=$ $(0,-1)$ is not identically negative when $1<\alpha \leq 2$, which leads to the instability of the difference method to fractional diffusion equations for the same reason in the stability analysis in Sections 3 and 4 .

2.2. Third order approximations. Similar to the second order approximations for Riemann-Liouville fractional derivatives, we give a combination of three shifted Grünwald difference operators

$$
{ }_{L} \mathcal{G}_{h, p, q, r}^{\alpha} u(x)=\lambda_{1} A_{h, p}^{\alpha} u(x)+\lambda_{2} A_{h, q}^{\alpha} u(x)+\lambda_{3} A_{h, r}^{\alpha} u(x),
$$

where $p, q, r$ are integers and mutually non-equal, and

$$
\begin{aligned}
& \lambda_{1}=\frac{12 q r-(6 q+6 r+1) \alpha+3 \alpha^{2}}{12\left(q r-p q-p r+p^{2}\right)}, \\
& \lambda_{2}=\frac{12 p r-(6 p+6 r+1) \alpha+3 \alpha^{2}}{12\left(p r-p q-q r+q^{2}\right)}, \\
& \lambda_{3}=\frac{12 p q-(6 p+6 q+1) \alpha+3 \alpha^{2}}{12\left(p q-p r-q r+r^{2}\right)} .
\end{aligned}
$$

Assuming $u \in L^{1}(\mathbb{R})$, and taking Fourier transform on (2.23), we get

$$
\begin{aligned}
\mathscr{F}\left[{ }_{L} \mathcal{G}_{h, p, q, r}^{\alpha} u\right](\omega) & =(i \omega)^{\alpha}\left(\lambda_{1} W_{p}(i \omega h)+\lambda_{2} W_{q}(i \omega h)+\lambda_{3} W_{r}(i \omega h)\right) \hat{u}(\omega) \\
& =(i \omega)^{\alpha}\left(1+C(i \omega h)^{3}\right) \hat{u}(\omega)
\end{aligned}
$$

where $W_{s}(z)$ is defined in (2.10). If ${ }_{-\infty} D_{x}^{\alpha+3} u$ and its Fourier transform belong to $L^{1}(\mathbb{R})$, then we have

$$
\begin{aligned}
\left|{ }_{L} \mathcal{G}_{h, p, q, r}^{\alpha} u-{ }_{-\infty} D_{x}^{\alpha} u\right| & \leq \frac{1}{2 \pi} \int_{\mathbb{R}}\left|\mathscr{F}\left[{ }_{L} \mathcal{G}_{h, p, q, r}^{\alpha} u-{ }_{-\infty} D_{x}^{\alpha} u\right]\right| \\
& \leq C\left\|\mathscr{F}\left[{ }_{-\infty} D_{x}^{\alpha+3} u\right](\omega)\right\|_{L^{1}} h^{3}=O\left(h^{3}\right) .
\end{aligned}
$$

The above results can be stated in the following theorem.

Theorem 2.16. Let $u \in L^{1}(\mathbb{R}),{ }_{-\infty} D_{x}^{\alpha+3} u$ and its Fourier transform belong to $L^{1}(\mathbb{R})$, and the following 3-WSGD operator (2.23) satisfies

$$
{ }_{L} \mathcal{G}_{h, p, q, r}^{\alpha} u(x)={ }_{-\infty} D_{x}^{\alpha} u(x)+O\left(h^{3}\right),
$$

uniformly for $x \in \mathbb{R}$.

If $u \in L^{1}(\mathbb{R}),{ }_{x} D_{\infty}^{\alpha+3} u$ and its Fourier transform belong to $L^{1}(\mathbb{R})$, we also have

$$
{ }_{R} \mathcal{G}_{h, p, q, r}^{\alpha} u(x)=\lambda_{1} B_{h, p}^{\alpha} u(x)+\lambda_{2} B_{h, q}^{\alpha} u(x)+\lambda_{3} B_{h, r}^{\alpha} u(x)={ }_{x} D_{\infty}^{\alpha} u+O\left(h^{3}\right),
$$

uniformly for $x \in \mathbb{R}$, where the operator $B_{h, s}^{\alpha}$ is given by (2.14), and $\lambda_{i}, i=1,2,3$ are the same as (2.24). 
As stated in Remark 2.7, the 3-WSGD operator can be utilized for approximating Riemann-Liouville fractional differential equations on bounded domain by the finite difference method when choosing $(p, q, r)=(1,0,-1)$, then the corresponding weight coefficients in (2.24) are $\lambda_{1}=\frac{5}{24} \alpha+\frac{1}{8} \alpha^{2}, \lambda_{2}=1+\frac{1}{12} \alpha-\frac{1}{4} \alpha^{2}$, $\lambda_{3}=-\frac{7}{24} \alpha+\frac{1}{8} \alpha^{2}$. For function $u(x)$ satisfying $u(a)=u(b)=0$ on grid points $\left\{x_{k}=a+k h, h=(b-a) / n, k=1, \ldots, n-1\right\}$, the approximation matrix of (2.23) with $(p, q, r)=(1,0,-1)$ is

$$
\begin{aligned}
& G=\lambda_{1}\left(\begin{array}{ccccc}
g_{1}^{(\alpha)} & g_{0}^{(\alpha)} & & & \\
g_{2}^{(\alpha)} & g_{1}^{(\alpha)} & g_{0}^{(\alpha)} & & \\
\vdots & g_{2}^{(\alpha)} & g_{1}^{(\alpha)} & \ddots & \\
g_{n-2}^{(\alpha)} & \ldots & \ddots & \ddots & g_{0}^{(\alpha)} \\
g_{n-1}^{(\alpha)} & g_{n-2}^{(\alpha)} & \cdots & g_{2}^{(\alpha)} & g_{1}^{(\alpha)}
\end{array}\right)+\lambda_{2}\left(\begin{array}{ccccc}
g_{0}^{(\alpha)} & & & & \\
g_{1}^{(\alpha)} & g_{0}^{(\alpha)} & & & \\
\vdots & g_{1}^{(\alpha)} & g_{0}^{(\alpha)} & & \\
g_{n-3}^{(\alpha)} & \cdots & \ddots & \ddots & \\
g_{n-2}^{(\alpha)} & g_{n-3}^{(\alpha)} & \cdots & g_{1}^{(\alpha)} & g_{0}^{(\alpha)}
\end{array}\right) \\
& +\lambda_{3}\left(\begin{array}{ccccc}
0 & & & & \\
g_{0}^{(\alpha)} & 0 & & & \\
\vdots & g_{0}^{(\alpha)} & 0 & & \\
g_{n-4}^{(\alpha)} & \cdots & \ddots & \ddots & \\
g_{n-3}^{(\alpha)} & g_{n-4}^{(\alpha)} & \cdots & g_{0}^{(\alpha)} & 0
\end{array}\right)
\end{aligned}
$$

Example 2.17. We utilize the approximation (2.23) for simulating the steady state fractional diffusion problem

$$
{ }_{-}{ }_{0} D_{x}^{\alpha} u(x)=-\frac{\Gamma(3+\alpha)}{2} x^{2}, \quad x \in(0,1),
$$

with $u(0)=0, u(1)=1$, and $1<\alpha<2$. The exact solution is $u(x)=x^{2+\alpha}$.

The 3-WSGD operator with $(p, q, r)=(1,0,-1)$ is utilized for computing the solution of Example 2.17, the numerical results are given in Table 1, from which the order and accuracy of the 3-WSGD operator is verified.

TABLE 1. The maximum and $L^{2}$ errors and their convergence rates to Example 2.17 approximated by the 3-WSGD operator for $\alpha=$ $1.1,1.9$.

\begin{tabular}{rcccccccc}
\hline & \multicolumn{9}{c}{$\alpha=1.1$} & & \multicolumn{3}{c}{$\alpha=1.9$} \\
\cline { 2 - 9 }$N$ & $\left\|u^{n}-U^{n}\right\|_{\infty}$ & rate & $\left\|u^{n}-U^{n}\right\|$ & rate & $\left\|u^{n}-U^{n}\right\|_{\infty}$ & rate & $\left\|u^{n}-U^{n}\right\|$ & rate \\
\hline 8 & $9.48629 \mathrm{E}-04$ & - & $5.92003 \mathrm{E}-04$ & - & $3.20333 \mathrm{E}-04$ & - & $1.59788 \mathrm{E}-04$ & - \\
16 & $1.19530 \mathrm{E}-04$ & 2.99 & $7.51799 \mathrm{E}-05$ & 2.98 & $2.29262 \mathrm{E}-05$ & 3.80 & $1.04858 \mathrm{E}-05$ & 3.93 \\
32 & $1.50130 \mathrm{E}-05$ & 2.99 & $9.47995 \mathrm{E}-06$ & 2.99 & $1.58500 \mathrm{E}-06$ & 3.85 & $6.71546 \mathrm{E}-07$ & 3.96 \\
64 & $1.88094 \mathrm{E}-06$ & 3.00 & $1.18999 \mathrm{E}-06$ & 2.99 & $1.07818 \mathrm{E}-07$ & 3.88 & $4.24776 \mathrm{E}-08$ & 3.98 \\
128 & $2.35382 \mathrm{E}-07$ & 3.00 & $1.49052 \mathrm{E}-07$ & 3.00 & $7.27733 \mathrm{E}-09$ & 3.89 & $2.67067 \mathrm{E}-09$ & 3.99 \\
256 & $2.94392 \mathrm{E}-08$ & 3.00 & $1.86501 \mathrm{E}-08$ & 3.00 & $4.89318 \mathrm{E}-10$ & 3.89 & $1.67325 \mathrm{E}-10$ & 4.00 \\
\hline
\end{tabular}


As in the above, the generating function of the symmetric part $\frac{G+G^{T}}{2}$ of the Toeplitz matrix $G$ is

$$
\begin{aligned}
f(\alpha ; x)= & \left(\frac{5}{48} \alpha+\frac{1}{16} \alpha^{2}\right)\left(\mathrm{e}^{-i x}\left(1-\mathrm{e}^{i x}\right)^{\alpha}+\mathrm{e}^{i x}\left(1-\mathrm{e}^{-i x}\right)^{\alpha}\right) \\
& +\left(\frac{1}{2}+\frac{1}{24} \alpha-\frac{1}{8} \alpha^{2}\right)\left(\left(1-\mathrm{e}^{i x}\right)^{\alpha}+\left(1-\mathrm{e}^{-i x}\right)^{\alpha}\right) \\
& +\left(-\frac{7}{48} \alpha+\frac{1}{16} \alpha^{2}\right)\left(\mathrm{e}^{i x}\left(1-\mathrm{e}^{i x}\right)^{\alpha}+\mathrm{e}^{-i x}\left(1-\mathrm{e}^{-i x}\right)^{\alpha}\right),
\end{aligned}
$$

$x \in[-\pi, \pi]$. As matrix $\frac{G+G^{T}}{2}$ is symmetric, thus $f(\alpha ; x)$ is a real-valued and even function, so we consider it on $[0, \pi]$ and get

$$
\begin{aligned}
f(\alpha ; x)= & \left(2 \sin \left(\frac{x}{2}\right)\right)^{\alpha}\left(\left(\frac{5}{48} \alpha+\frac{1}{16} \alpha^{2}\right) \cos \left(\frac{\alpha}{2}(x-\pi)-x\right)\right. \\
& +\left(\frac{1}{2}+\frac{1}{24} \alpha-\frac{1}{8} \alpha^{2}\right) \cos \left(\frac{\alpha}{2}(x-\pi)\right) \\
& \left.+\left(-\frac{7}{48} \alpha+\frac{1}{16} \alpha^{2}\right) \cos \left(\frac{\alpha}{2}(x-\pi)+x\right)\right) .
\end{aligned}
$$

We can check that $f(\alpha ; x)$ is identically negative only for $\frac{1+\sqrt{73}}{6}<\alpha \leq 2$, then the real parts of the eigenvalues of matrix $G$ are not always negative when $1<\alpha \leq 2$, so the finite difference scheme using (2.23) or (2.28) for time dependent fractional problems will not be unconditionally stable for $1<\alpha<\frac{1+\sqrt{73}}{6}$.

\section{ONE-DIMENSIONAL SPACE FRACTIONAL DIFFUSION EQUATION}

In this section, we consider the following two-sided one-dimensional space fractional diffusion equation

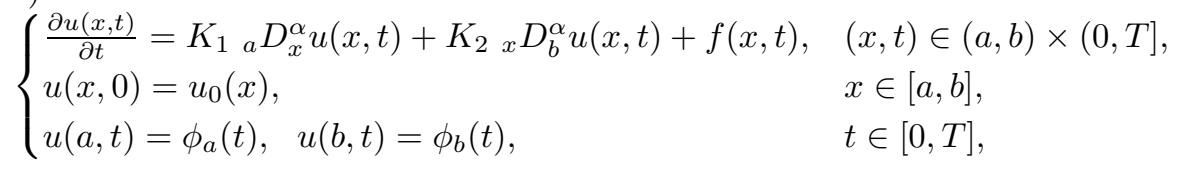

where both ${ }_{a} D_{x}^{\alpha}$ and ${ }_{x} D_{b}^{\alpha}$ are Riemann-Liouville fractional operators with $1<\alpha \leq 2$. The diffusion coefficients $K_{1}$ and $K_{2}$ are non-negative constants with $K_{1}^{2}+K_{2}^{2} \neq 0$; if $K_{1} \neq 0$, then $\phi_{a}(t) \equiv 0$; if $K_{2} \neq 0$, then $\phi_{b}(t) \equiv 0$. Next we will discretize the problem (3.1) by the second order accurate WSGD formulae (2.16). In the analysis of the numerical method that follows, we assume that (3.1) has a unique and sufficiently smooth solution.

3.1. CN-WSGD scheme. We partition the interval $[a, b]$ into a uniform mesh with the space step $h=(b-a) / N$ and the time step $\tau=T / M$, where $N, M$ are two positive integers, and the set of grid points is denoted by $x_{i}=i h$ and $t_{n}=n \tau$ for $0 \leq i \leq N$ and $0 \leq n \leq M$. Let $t_{n+1 / 2}=\left(t_{n}+t_{n+1}\right) / 2$ for $0 \leq n \leq M-1$, and we use the following notations:

$$
u_{i}^{n}=u\left(x_{i}, t_{n}\right), \quad f_{i}^{n+1 / 2}=f\left(x_{i}, t_{n+1 / 2}\right), \quad \delta_{t} u_{i}^{n}=\left(u_{i}^{n+1}-u_{i}^{n}\right) / \tau .
$$

Using the Crank-Nicolson technique for the time discretization of (3.1) leads to $\delta_{t} u_{i}^{n}-\frac{1}{2}\left(K_{1}\left({ }_{a} D_{x}^{\alpha} u\right)_{i}^{n}+K_{1}\left({ }_{a} D_{x}^{\alpha} u\right)_{i}^{n+1}+K_{2}\left({ }_{x} D_{b}^{\alpha} u\right)_{i}^{n}+K_{2}\left({ }_{x} D_{b}^{\alpha} u\right)_{i}^{n+1}\right)=f_{i}^{n+1 / 2}+O\left(\tau^{2}\right)$. 
In space discretization, we choose the WSGD operators ${ }_{L} \mathcal{D}_{h, p, q}^{\alpha} u(x, t)$ and ${ }_{R} \mathcal{D}_{h, p, q}^{\alpha} u(x, t)$ to approximate the Riemann-Liouville fractional derivatives ${ }_{a} D_{x}^{\alpha} u(x, t)$ and ${ }_{x} D_{b}^{\alpha} u(x, t)$ with second order accuracy, respectively, and $(p, q)=$ $(1,0)$ or $(1,-1)$. This implies that

$$
\begin{aligned}
& \delta_{t} u_{i}^{n}-\frac{1}{2}\left(K_{1{ }_{L}} \mathcal{D}_{h, p, q}^{\alpha} u_{i}^{n}+K_{1{ }_{L}} \mathcal{D}_{h, p, q}^{\alpha} u_{i}^{n+1}+K_{2}{ }_{R} \mathcal{D}_{h, p, q}^{\alpha} u_{i}^{n}+K_{2}{ }_{R} \mathcal{D}_{h, p, q}^{\alpha} u_{i}^{n+1}\right) \\
& =f_{i}^{n+1 / 2}+\varepsilon_{i}^{n},
\end{aligned}
$$

where

$$
\left|\varepsilon_{i}^{n}\right| \leq \tilde{c}\left(\tau^{2}+h^{2}\right) .
$$

Multiplying (3.2) by $\tau$ and separating the time layers, we have

$$
\begin{aligned}
& u_{i}^{n+1}-\frac{K_{1} \tau}{2}{ }_{L} \mathcal{D}_{h, p, q}^{\alpha} u_{i}^{n+1}-\frac{K_{2} \tau}{2}{ }_{R} \mathcal{D}_{h, p, q}^{\alpha} u_{i}^{n+1} \\
& =u_{i}^{n}+\frac{K_{1} \tau}{2}{ }_{L} \mathcal{D}_{h, p, q}^{\alpha} u_{i}^{n}+\frac{K_{2} \tau}{2}{ }_{R} \mathcal{D}_{h, p, q}^{\alpha} u_{i}^{n}+\tau f_{i}^{n+1 / 2}+\tau \varepsilon_{i}^{n} .
\end{aligned}
$$

Substituting ${ }_{L} \mathcal{D}_{h, p, q}^{\alpha} u,{ }_{R} \mathcal{D}_{h, p, q}^{\alpha} u$ by (2.16), we obtain that

$$
\begin{aligned}
& u_{i}^{n+1}-\frac{K_{1} \tau}{2 h^{\alpha}} \sum_{k=0}^{i+1} w_{k}^{(\alpha)} u_{i-k+1}^{n+1}-\frac{K_{2} \tau}{2 h^{\alpha}} \sum_{k=0}^{N-i+1} w_{k}^{(\alpha)} u_{i+k-1}^{n+1} \\
& =u_{i}^{n}+\frac{K_{1} \tau}{2 h^{\alpha}} \sum_{k=0}^{i+1} w_{k}^{(\alpha)} u_{i-k+1}^{n}+\frac{K_{2} \tau}{2 h^{\alpha}} \sum_{k=0}^{N-i+1} w_{k}^{(\alpha)} u_{i+k-1}^{n}+\tau f_{i}^{n+1 / 2}+\tau \varepsilon_{i}^{n} .
\end{aligned}
$$

Denoting $U_{i}^{n}$ as the numerical approximation of $u_{i}^{n}$, we derive the CN-WSGD scheme for (3.1):

$$
\begin{aligned}
& U_{i}^{n+1}-\frac{K_{1} \tau}{2 h^{\alpha}} \sum_{k=0}^{i+1} w_{k}^{(\alpha)} U_{i-k+1}^{n+1}-\frac{K_{2} \tau}{2 h^{\alpha}} \sum_{k=0}^{N-i+1} w_{k}^{(\alpha)} U_{i+k-1}^{n+1} \\
& =U_{i}^{n}+\frac{K_{1} \tau}{2 h^{\alpha}} \sum_{k=0}^{i+1} w_{k}^{(\alpha)} U_{i-k+1}^{n}+\frac{K_{2} \tau}{2 h^{\alpha}} \sum_{k=0}^{N-i+1} w_{k}^{(\alpha)} U_{i+k-1}^{n}+\tau f_{i}^{n+1 / 2} .
\end{aligned}
$$

For the convenience of implementation, using the matrix form of the grid functions

$$
U^{n}=\left(U_{1}^{n}, U_{2}^{n}, \cdots, U_{N-1}^{n}\right)^{\mathrm{T}}, \quad F^{n}=\left(f_{1}^{n+1 / 2}, f_{2}^{n+1 / 2}, \cdots, f_{N-1}^{n+1 / 2}\right)^{\mathrm{T}},
$$

makes the finite difference scheme (3.6) be described as

$$
\left(I-\frac{\tau}{2 h^{\alpha}}\left(K_{1} A+K_{2} A^{\mathrm{T}}\right)\right) U^{n+1}=\left(I+\frac{\tau}{2 h^{\alpha}}\left(K_{1} A+K_{2} A^{\mathrm{T}}\right)\right) U^{n}+\tau F^{n}+H^{n}
$$

where $A$ is given by (2.20) and

$$
H^{n}=\frac{\tau}{2 h^{\alpha}}\left[\begin{array}{c}
K_{1} w_{2}^{(\alpha)}+K_{2} w_{0}^{(\alpha)} \\
K_{1} w_{3}^{(\alpha)} \\
\vdots \\
K_{1} w_{N-1}^{(\alpha)} \\
K_{1} w_{N}^{(\alpha)}
\end{array}\right]\left(U_{0}^{n}+U_{0}^{n+1}\right)+\frac{\tau}{2 h^{\alpha}}\left[\begin{array}{c}
K_{2} w_{N}^{(\alpha)} \\
K_{2} w_{N-1}^{(\alpha)} \\
\vdots \\
K_{2} w_{3}^{(\alpha)} \\
K_{1} w_{0}^{(\alpha)}+K_{2} w_{2}^{(\alpha)}
\end{array}\right]\left(U_{N}^{n}+U_{N}^{n+1}\right) .
$$


3.2. Stability and convergence. Now we consider the stability and convergence analysis for the CN-WSGD scheme (3.7). Define

$$
V_{h}=\left\{v: v=\left\{v_{i}\right\} \text { is a grid function in }\left\{x_{i}=i h\right\}_{i=0}^{N} \text { and } v_{0}=v_{N}=0\right\} .
$$

For any $v=\left\{v_{i}\right\} \in V_{h}$, we define its pointwise maximum norm

$$
\|v\|_{\infty}=\max _{1 \leq i \leq N-1}\left|v_{i}\right|
$$

and the following discrete $L^{2}$-norm

$$
\|v\|=\sqrt{h \sum_{i=1}^{N-1} v_{i}^{2} .}
$$

Theorem 3.1. The finite difference scheme (3.6) is unconditionally stable.

Proof. Denoting $B=\frac{\tau}{2 h^{\alpha}}\left(K_{1} A+K_{2} A^{\mathrm{T}}\right)$, the matrix form of the difference approximation for problem (3.1) can be rewritten as

$$
(I-B) U^{n+1}=(I+B) U^{n}+\tau F^{n}+H^{n},
$$

and the relationship between the error $e^{n+1}$ in $U^{n+1}$ and the error $e^{n}$ in $U^{n}$ is given by

$$
e^{n+1}=(I-B)^{-1}(I+B) e^{n} .
$$

If we denote $\lambda$ as an eigenvalue of matrix $B$, then $\frac{1+\lambda}{1-\lambda}$ is the eigenvalue of matrix $(I-B)^{-1}(I+B)$. The result of Theorem 2.13 shows that the eigenvalues of matrix $\frac{B+B^{\mathrm{T}}}{2}=\frac{\tau\left(K_{1}+K_{2}\right)}{4 h^{\alpha}}\left(A+A^{\mathrm{T}}\right)$ are negative, thus $\operatorname{Re}(\lambda)<0$, which implies that $\left|\frac{1+\lambda}{1-\lambda}\right|<1$. Therefore, the spectral radius of matrix $(I-B)^{-1}(I+B)$ is less than one, which yields that $\left((I-B)^{-1}(I+B)\right)^{n}$ converges to zero matrix (see Theorem 1.5 in [21]). Then the difference scheme (3.6) is unconditionally stable.

Remark 3.2. Considering the $\theta$ weighted scheme for the time discretization of (3.1), then the iterative matrix of the full discrete scheme is

$$
(I-\theta B)^{-1}(I+(1-\theta) B) \text {. }
$$

If $\lambda$ is an eigenvalue of matrix $B$, then the eigenvalue of (3.12) is $\frac{1+(1-\theta) \lambda}{1-\theta \lambda}$. As $\operatorname{Re}(\lambda)<0$, it is easy to check that

$$
\left|\frac{1+(1-\theta) \lambda}{1-\theta \lambda}\right|<1
$$

for $\frac{1}{2} \leq \theta \leq 1$. Then the $\theta$ weighted WSGD scheme for (3.1) is unconditionally stable when $\frac{1}{2} \leq \theta \leq 1$.

The following theorem shows the unconditional convergence of the scheme (3.6).

Theorem 3.3. Let $u_{i}^{n}$ be the exact solution of problem (3.1), and $U_{i}^{n}$ the solution of the finite difference scheme (3.6), then for all $1 \leq n \leq M$, we have

$$
\left\|u^{n}-U^{n}\right\| \leq c\left(\tau^{2}+h^{2}\right),
$$

where $c$ denotes a positive constant and $\|\cdot\|$ stands for the discrete $L^{2}$-norm. 
Proof. Let $e_{i}^{n}=u_{i}^{n}-U_{i}^{n}$, and from (3.5) and (3.6) we have

$$
\left(e^{n+1}-e^{n}\right)-\frac{K_{1} \tau}{2 h^{\alpha}} A\left(e^{n+1}+e^{n}\right)-\frac{K_{2} \tau}{2 h^{\alpha}} A^{\mathrm{T}}\left(e^{n+1}+e^{n}\right)=\tau \varepsilon^{n},
$$

where

$$
e^{n}=\left(u_{1}^{n}-U_{1}^{n}, u_{2}^{n}-U_{2}^{n}, \cdots, u_{N-1}^{n}-U_{N-1}^{n}\right)^{\mathrm{T}}, \varepsilon^{n}=\left(\varepsilon_{1}^{n}, \varepsilon_{2}^{n}, \cdots, \varepsilon_{N-1}^{n}\right)^{\mathrm{T}} .
$$

Multiplying (3.15) by $h$, and acting $\left(e^{n+1}+e^{n}\right)^{\mathrm{T}}$ on both sides, we obtain that

$$
\begin{array}{r}
h\left(e^{n+1}+e^{n}\right)^{\mathrm{T}} I\left(e^{n+1}-e^{n}\right)-\frac{K_{1} \tau}{2 h^{\alpha-1}}\left(e^{n+1}+e^{n}\right)^{\mathrm{T}} A\left(e^{n+1}+e^{n}\right) \\
-\frac{K_{2} \tau}{2 h^{\alpha-1}}\left(e^{n+1}+e^{n}\right)^{\mathrm{T}} A^{\mathrm{T}}\left(e^{n+1}+e^{n}\right)=\tau h\left(e^{n+1}+e^{n}\right)^{\mathrm{T}} \varepsilon^{n} .
\end{array}
$$

By Theorem 2.13. $A$ and its transpose $A^{\mathrm{T}}$ both being negative definite matrices, we get

$$
\left(e^{n+1}+e^{n}\right)^{\mathrm{T}} A\left(e^{n+1}+e^{n}\right)<0, \quad\left(e^{n+1}+e^{n}\right)^{\mathrm{T}} A^{\mathrm{T}}\left(e^{n+1}+e^{n}\right)<0,
$$

and from (3.16) and (3.17) it yields that

$$
\left\|e^{n+1}\right\|^{2}-\left\|e^{n}\right\|^{2} \leq \tau h\left(e^{n+1}+e^{n}\right)^{\mathrm{T}} \varepsilon^{n} \leq \tau\left(\left\|e^{n+1}\right\|+\left\|e^{n}\right\|\right) \cdot\left\|\varepsilon^{n}\right\| .
$$

Then we have

$$
\left\|e^{n+1}\right\|-\left\|e^{n}\right\| \leq \tau\left\|\varepsilon^{n}\right\|, \quad n \geq 0
$$

Consequently,

$$
\left\|e^{n}\right\| \leq \tau \sum_{k=1}^{n}\left\|\varepsilon^{n}\right\| \leq c\left(\tau^{2}+h^{2}\right), \quad n \geq 1,
$$

which is the result that we need.

\section{TWO-DIMENSIONAL SPACE FRACTIONAL DIFFUSION EQUATION}

We next consider the following two-sided space fractional diffusion equation in two dimensions

$$
\begin{cases}\frac{\partial u(x, y, t)}{\partial t}=\left(K_{1}^{+}{ }_{a} D_{x}^{\alpha} u(x, y, t)+K_{2}^{+}{ }_{x} D_{b}^{\alpha} u(x, y, t)\right) & \\ +\left(K_{1}^{-}{ }_{c} D_{y}^{\beta} u(x, y, t)+K_{2}^{-}{ }_{y} D_{d}^{\beta} u(x, y, t)\right)+f(x, y, t), & (x, y, t) \in \Omega \times[0, T], \\ u(x, y, 0)=u_{0}(x, y), & (x, y) \in \Omega, \\ u(x, y, t)=\varphi(x, y, t), & (x, y, t) \in \partial \Omega \times[0, T],\end{cases}
$$

where $\Omega=(a, b) \times(c, d),{ }_{a} D_{x}^{\alpha},{ }_{x} D_{b}^{\alpha}$ and ${ }_{c} D_{y}^{\beta},{ }_{y} D_{d}^{\beta}$ are Riemann-Liouville fractional operators with $1<\alpha, \beta \leq 2$. The diffusion coefficients satisfy $K_{i}^{+}, K_{i}^{-} \geq 0, i=$ $1,2,\left(K_{1}^{+}\right)^{2}+\left(K_{2}^{+}\right)^{2} \neq 0$ and $\left(K_{1}^{-}\right)^{2}+\left(K_{2}^{-}\right)^{2} \neq 0$, and the boundary function $\varphi$ satisfies, if $K_{1}^{+} \neq 0$, then $\varphi(a, y, t)=0$; if $K_{2}^{+} \neq 0$, then $\varphi(b, y, t)=0$; if $K_{1}^{-} \neq 0$, then $\varphi(x, c, t)=0$; if $K_{2}^{-} \neq 0$, then $\varphi(x, d, t)=0$. We assume that (4.1) has a unique and sufficiently smooth solution. 
4.1. CN-WSGD scheme. Now we establish the Crank-Nicolson difference scheme by using WSGD formulae (2.16) for problem (4.1). We partition the domain $\Omega$ into a uniform mesh with the space steps $h_{x}=(b-a) / N_{x}, h_{y}=(d-c) / N_{y}$ and the time step $\tau=T / M$, where $N_{x}, N_{y}, M$ being positive integers, and the set of grid points is denoted by $x_{i}=i h_{x}, y_{j}=j h_{y}$ and $t_{n}=n \tau$ for $0 \leq i \leq N_{x}, 0 \leq j \leq N_{y}$ and $0 \leq n \leq M$. Let $t_{n+1 / 2}=\left(t_{n}+t_{n+1}\right) / 2$ for $0 \leq n \leq M-1$, and we use the following notations:

$$
u_{i, j}^{n}=u\left(x_{i}, y_{j}, t_{n}\right), \quad f_{i, j}^{n+1 / 2}=f\left(x_{i}, y_{j}, t_{n+1 / 2}\right), \quad \delta_{t} u_{i, j}^{n}=\left(u_{i, j}^{n+1}-u_{i, j}^{n}\right) / \tau .
$$

Discretizing (4.1) in time direction leads to

$$
\begin{aligned}
\delta_{t} u_{i, j}^{n}= & \frac{1}{2}\left(K_{1}^{+}\left({ }_{a} D_{x}^{\alpha} u\right)_{i, j}^{n+1}+K_{2}^{+}\left({ }_{x} D_{b}^{\alpha} u\right)_{i, j}^{n+1}+K_{1}^{-}\left({ }_{c} D_{y}^{\beta} u\right)_{i, j}^{n+1}+K_{2}^{-}\left({ }_{y} D_{d}^{\beta} u\right)_{i, j}^{n+1}\right. \\
& \left.+K_{1}^{+}\left({ }_{a} D_{x}^{\alpha} u\right)_{i, j}^{n}+K_{2}^{+}\left({ }_{x} D_{b}^{\alpha} u\right)_{i, j}^{n}+K_{1}^{-}\left({ }_{c} D_{y}^{\beta} u\right)_{i, j}^{n}+K_{2}^{-}\left({ }_{y} D_{d}^{\beta} u\right)_{i, j}^{n}\right) \\
& +f_{i, j}^{n+1 / 2}+O\left(\tau^{2}\right) .
\end{aligned}
$$

In space discretization, we choose the WSGD operators ${ }_{L} \mathcal{D}_{h_{x}, p, q}^{\alpha} u,{ }_{R} \mathcal{D}_{h_{x}, p, q}^{\alpha} u$ and ${ }_{L} \mathcal{D}_{h_{y}, p, q}^{\beta} u,{ }_{R} \mathcal{D}_{h_{y}, p, q}^{\beta} u$ to respectively approximate the fractional diffusion terms ${ }_{a} D_{x}^{\alpha} u,{ }_{x} D_{b}^{\alpha} u$ and ${ }_{c} D_{y}^{\beta} u,{ }_{y} D_{d}^{\beta} u$, and multiplying (4.2) by $\tau$ and separating the time layers, we have that

$$
\begin{aligned}
& \left(1-\frac{K_{1}^{+} \tau}{2}{ }_{L} \mathcal{D}_{h_{x}, p, q}^{\alpha}-\frac{K_{2}^{+} \tau}{2}{ }_{R} \mathcal{D}_{h_{x}, p, q}^{\alpha}-\frac{K_{1}^{-} \tau}{2}{ }_{L} \mathcal{D}_{h_{y}, p, q}^{\beta}-\frac{K_{2}^{-} \tau}{2}{ }_{R} \mathcal{D}_{h_{y}, p, q}^{\beta}\right) u_{i, j}^{n+1} \\
& =\left(1+\frac{K_{1}^{+} \tau}{2}{ }_{L} \mathcal{D}_{h_{x}, p, q}^{\alpha}+\frac{K_{2}^{+} \tau}{2}{ }_{R} \mathcal{D}_{h_{x}, p, q}^{\alpha}+\frac{K_{1}^{-} \tau}{2}{ }_{L} \mathcal{D}_{h_{y}, p, q}^{\beta}+\frac{K_{2}^{-} \tau}{2}{ }_{R} \mathcal{D}_{h_{y}, p, q}^{\beta}\right) u_{i, j}^{n} \\
& \quad+\tau f_{i, j}^{n+1 / 2}+\tau \hat{\varepsilon}_{i, j}^{n},
\end{aligned}
$$

where $\left|\hat{\varepsilon}_{i, j}^{n}\right| \leq \tilde{c}\left(\tau^{2}+h_{x}^{2}+h_{y}^{2}\right)$ denotes the truncation error. We denote

$$
\delta_{x}^{\alpha}=K_{1}^{+}{ }_{L} \mathcal{D}_{h_{x}, p, q}^{\alpha}+K_{2}^{+}{ }_{R} \mathcal{D}_{h_{x}, p, q}^{\alpha}, \quad \delta_{y}^{\beta}=K_{1}^{-}{ }_{L} \mathcal{D}_{h_{y}, p, q}^{\beta}+K_{2}^{-}{ }_{R} \mathcal{D}_{h_{y}, p, q}^{\beta} .
$$

Using the Taylor expansion, we have

$$
\begin{aligned}
\frac{\tau^{2}}{4} \delta_{x}^{\alpha} \delta_{y}^{\beta}\left(u_{i, j}^{n+1}-u_{i, j}^{n}\right)= & \frac{\tau^{3}}{4}\left(\left(K_{1}^{+}{ }_{a} D_{x}^{\alpha}+K_{2}^{+}{ }_{x} D_{b}^{\alpha}\right)\left(K_{1}^{-}{ }_{c} D_{y}^{\beta}+K_{2}^{-}{ }_{y} D_{d}^{\beta}\right) u_{t}\right)_{i, j}^{n+1 / 2} \\
& +\tau^{3} O\left(\tau^{2}+h_{x}^{2}+h_{y}^{2}\right) .
\end{aligned}
$$

Adding formula (4.4) to the right-hand side of (4.3) and making the factorization leads to

$$
\left(1-\frac{\tau}{2} \delta_{x}^{\alpha}\right)\left(1-\frac{\tau}{2} \delta_{y}^{\beta}\right) u_{i, j}^{n+1}=\left(1+\frac{\tau}{2} \delta_{x}^{\alpha}\right)\left(1+\frac{\tau}{2} \delta_{y}^{\beta}\right) u_{i, j}^{n}+\tau f_{i, j}^{n+1 / 2}+\tau \varepsilon_{i, j}^{n},
$$

where $\varepsilon_{i, j}^{n}=\hat{\varepsilon}_{i, j}^{n}+O\left(\tau^{2}\right)$. Denoting by $U_{i, j}^{n}$ the numerical approximation to $u_{i, j}^{n}$, we obtain the finite difference approximation for problem (4.1):

$$
\left(1-\frac{\tau}{2} \delta_{x}^{\alpha}\right)\left(1-\frac{\tau}{2} \delta_{y}^{\beta}\right) U_{i, j}^{n+1}=\left(1+\frac{\tau}{2} \delta_{x}^{\alpha}\right)\left(1+\frac{\tau}{2} \delta_{y}^{\beta}\right) U_{i, j}^{n}+\tau f_{i, j}^{n+1 / 2}
$$


For efficiently solving (4.6), the following techniques can be used. Peaceman-Rachford ADI scheme [18, 25]:

$$
\begin{aligned}
& \left(1-\frac{\tau}{2} \delta_{x}^{\alpha}\right) V_{i, j}^{n}=\left(1+\frac{\tau}{2} \delta_{y}^{\beta}\right) U_{i, j}^{n}+\frac{\tau}{2} f_{i, j}^{n+1 / 2}, \\
& \left(1-\frac{\tau}{2} \delta_{y}^{\beta}\right) U_{i, j}^{n+1}=\left(1+\frac{\tau}{2} \delta_{x}^{\alpha}\right) V_{i, j}^{n}+\frac{\tau}{2} f_{i, j}^{n+1 / 2}
\end{aligned}
$$

Douglas ADI scheme [7]:

$$
\begin{aligned}
& \left(1-\frac{\tau}{2} \delta_{x}^{\alpha}\right) V_{i, j}^{n}=\left(1+\frac{\tau}{2} \delta_{x}^{\alpha}+\tau \delta_{y}^{\beta}\right) U_{i, j}^{n}+\tau f_{i, j}^{n+1 / 2}, \\
& \left(1-\frac{\tau}{2} \delta_{y}^{\beta}\right) U_{i, j}^{n+1}=V_{i, j}^{n}-\frac{\tau}{2} \delta_{y}^{\beta} U_{i, j}^{n} .
\end{aligned}
$$

D'Yakonov ADI scheme [25]:

$$
\begin{aligned}
& \left(1-\frac{\tau}{2} \delta_{x}^{\alpha}\right) V_{i, j}^{n}=\left(1+\frac{\tau}{2} \delta_{x}^{\alpha}\right)\left(1+\frac{\tau}{2} \delta_{y}^{\beta}\right) U_{i, j}^{n}+\tau f_{i, j}^{n+1 / 2}, \\
& \left(1-\frac{\tau}{2} \delta_{y}^{\beta}\right) U_{i, j}^{n+1}=V_{i, j}^{n} .
\end{aligned}
$$

A simple calculation shows that

$$
\frac{\tau^{3}}{4} \delta_{x}^{\alpha} \delta_{y}^{\beta} f_{i, j}^{n+1 / 2}=\frac{\tau^{3}}{4}\left(K_{1}^{+}{ }_{a} D_{x}^{\alpha}+K_{2}^{+}{ }_{x} D_{b}^{\alpha}\right)\left(K_{1}^{-}{ }_{c} D_{y}^{\beta}+K_{2}^{-}{ }_{y} D_{d}^{\beta}\right) f_{i, j}^{n+1 / 2}+\tau^{3} O\left(h_{x}^{2}+h_{y}^{2}\right) \text {. }
$$

Then from (4.5) and (4.10), it yields that

$$
\begin{aligned}
\left(1-\frac{\tau}{2} \delta_{x}^{\alpha}\right)\left(1-\frac{\tau}{2} \delta_{y}^{\beta}\right) u_{i, j}^{n+1}= & \left(1+\frac{\tau}{2} \delta_{x}^{\alpha}\right)\left(1+\frac{\tau}{2} \delta_{y}^{\beta}\right) u_{i, j}^{n} \\
& +\tau f_{i, j}^{n+1 / 2}+\frac{\tau^{3}}{4} \delta_{x}^{\alpha} \delta_{y}^{\beta} f_{i, j}^{n+1 / 2}+\tau \tilde{\varepsilon}_{i, j}^{n} .
\end{aligned}
$$

where

$$
\tilde{\varepsilon}_{i, j}^{n}=\varepsilon_{i, j}^{n}+O\left(\tau^{2} h_{x}^{2}+\tau^{2} h_{y}^{2}\right) .
$$

Eliminating the truncating error and denoting $U_{i, j}^{n}$ as the numerical approximation of $u_{i, j}^{n}$, we have

$$
\begin{aligned}
\left(1-\frac{\tau}{2} \delta_{x}^{\alpha}\right)\left(1-\frac{\tau}{2} \delta_{y}^{\beta}\right) U_{i, j}^{n+1}= & \left(1+\frac{\tau}{2} \delta_{x}^{\alpha}\right)\left(1+\frac{\tau}{2} \delta_{y}^{\beta}\right) U_{i, j}^{n} \\
& +\tau f_{i, j}^{n+1 / 2}+\frac{\tau^{3}}{4} \delta_{x}^{\alpha} \delta_{y}^{\beta} f_{i, j}^{n+1 / 2} .
\end{aligned}
$$

Introducing the intermediate variable $V_{i, j}^{n}$, we obtain the locally one-dimensional (LOD) scheme mentioned in [22,29],

$$
\begin{aligned}
& \left(1-\frac{\tau}{2} \delta_{x}^{\alpha}\right) V_{i, j}^{n}=\left(1+\frac{\tau}{2} \delta_{x}^{\alpha}\right) U_{i, j}^{n}+\frac{\tau}{2}\left(1+\frac{\tau}{2} \delta_{x}^{\alpha}\right) f_{i, j}^{n+1 / 2} \\
& \left(1-\frac{\tau}{2} \delta_{y}^{\beta}\right) U_{i, j}^{n+1}=\left(1+\frac{\tau}{2} \delta_{y}^{\beta}\right) V_{i, j}^{n}+\frac{\tau}{2}\left(1-\frac{\tau}{2} \delta_{y}^{\beta}\right) f_{i, j}^{n+1 / 2}
\end{aligned}
$$

4.2. Stability and convergence. Now we consider the stability and convergence analysis for the CN-WSGD scheme (4.6). Define the sets of the index of the interior and boundary mesh grid points in domain $[a, b] \times[c, d]$, respectively, as

$$
\begin{aligned}
& \Lambda_{h}=\left\{(i, j): 1 \leq i \leq N_{x}-1,1 \leq j \leq N_{y}-1\right\}, \\
& \partial \Lambda_{h}=\left\{(i, j): i=0, N_{x} ; 0 \leq j \leq N_{y}\right\} \cup\left\{(i, j): 1 \leq i \leq N_{x}-1 ; j=0, N_{y}\right\} .
\end{aligned}
$$


For any $v=\left\{v_{i}\right\} \in V_{h}$, we define its pointwise maximum norm and discrete $L^{2}$ norm, respectively, as

$$
\|v\|_{\infty}=\max _{(i, j) \in \Lambda_{h}}\left|v_{i, j}\right|, \quad\|v\|=\sqrt{h_{x} h_{y} \sum_{i=1}^{N_{x}-1} \sum_{j=1}^{N_{y}-1} v_{i, j}^{2}}
$$

where

$$
V_{h}=\left\{v: v=\left\{v_{i, j}\right\} \text { is a grid function in } \Lambda_{h} \cup \partial \Lambda_{h} \text { and } v_{i, j}=0 \text { on } \partial \Lambda_{h}\right\} .
$$

In the following, we list some properties of Kronecker products of matrices.

Lemma 4.1 ([11]). Let $A \in \mathbb{R}^{n \times n}$ have eigenvalues $\left\{\lambda_{i}\right\}_{i=1}^{n}$, and $B \in \mathbb{R}^{m \times m}$ have eigenvalues $\left\{\mu_{j}\right\}_{j=1}^{m}$. Then the $m n$ eigenvalues of $A \otimes B$, which represents the Kronecker product of matrix $A$ and $B$, are

$$
\lambda_{1} \mu_{1}, \ldots, \lambda_{1} \mu_{m}, \lambda_{2} \mu_{1}, \ldots, \lambda_{2} \mu_{m}, \ldots, \lambda_{n} \mu_{1}, \ldots, \lambda_{n} \mu_{m} .
$$

Lemma 4.2 ([11]). Let $A \in \mathbb{R}^{m \times n}, B \in \mathbb{R}^{r \times s}, C \in \mathbb{R}^{n \times p}, D \in \mathbb{R}^{s \times t}$. Then

$$
(A \otimes B)(C \otimes D)=A C \otimes B D\left(\in \mathbb{R}^{m r \times p t}\right) .
$$

Moreover, if $A, B \in \mathbb{R}^{n \times n}, I$ is a unit matrix of order $n$, then matrices $I \otimes A$ and $B \otimes I$ commute.

Lemma 4.3 ([1]). For all $A$ and $B,(A \otimes B)^{\mathrm{T}}=A^{\mathrm{T}} \otimes B^{\mathrm{T}}$.

The theoretical stability and convergence analysis of the designed numerical scheme for two-dimensional problem are given by the following theorems.

Theorem 4.4. The difference scheme (4.6) and (4.13) are unconditionally stable for $1<\alpha, \beta \leq 2$.

Proof. We represent the discrete functions $U_{i, j}^{n}$ and $f_{i, j}^{n+1 / 2}$ into vector forms with

$$
\begin{aligned}
U^{n}= & \left(u_{1,1}^{n}, u_{2,1}^{n}, \cdots, u_{N_{x}-1,1}^{n}, u_{1,2}^{n}, u_{2,2}^{n}, \cdots, u_{N_{x}-1,2}^{n}, \cdots,\right. \\
& \left.u_{1, N_{y}-1}^{n}, u_{2, N_{y}-1}^{n}, \cdots, u_{N_{x}-1, N_{y}-1}^{n}\right)^{\mathrm{T}}, \\
F^{n+1 / 2}= & \left(f_{1,1}^{n+1 / 2}, f_{2,1}^{n+1 / 2}, \cdots, f_{N_{x}-1,1}^{n+1 / 2}, f_{1,2}^{n+1 / 2}, f_{2,2}^{n+1 / 2}, \cdots, f_{N_{x}-1,2}^{n+1 / 2}, \cdots,\right. \\
& \left.f_{1, N_{y}-1}^{n+1 / 2}, f_{2, N_{y}-1}^{n+1 / 2}, \cdots, f_{N_{x}-1, N_{y}-1}^{n+1 / 2}\right)^{\mathrm{T}},
\end{aligned}
$$

and denote

$$
\mathcal{D}_{x}=\frac{K_{1}^{+} \tau}{2 h_{x}^{\alpha}} I_{y} \otimes A_{\alpha}+\frac{K_{2}^{+} \tau}{2 h_{x}^{\alpha}} I_{y} \otimes A_{\alpha}^{\mathrm{T}}, \mathcal{D}_{y}=\frac{K_{1}^{-} \tau}{2 h_{y}^{\beta}} A_{\beta} \otimes I_{x}+\frac{K_{2}^{-} \tau}{2 h_{y}^{\beta}} A_{\beta}^{\mathrm{T}} \otimes I_{x},
$$

where the symbol $\otimes$ denotes the Kronecker product, $I_{x}$ and $I_{y}$ are unit matrices of $\left(N_{x}-1\right)$ and $\left(N_{y}-1\right)$ squares, respectively, and matrices $A_{\alpha}$ and $A_{\beta}$ are defined in (2.20) corresponding to $\alpha, \beta$, respectively.

From the difference scheme (4.6) and (4.13), we have the relationship between the error $e^{n+1}$ in $U^{n+1}$ and the error $e^{n}$ in $U^{n}$ as

$$
e^{n+1}=\left(I-\mathcal{D}_{y}\right)^{-1}\left(I-\mathcal{D}_{x}\right)^{-1}\left(I+\mathcal{D}_{x}\right)\left(I+\mathcal{D}_{y}\right) e^{n},
$$

where $I$ is the unit matrix of $\left(N_{x}-1\right) \times\left(N_{y}-1\right)$ squares. Using Lemma 4.2 we can check that $\mathcal{D}_{x}$ and $\mathcal{D}_{y}$ commute, i.e.,

$$
\mathcal{D}_{x} \mathcal{D}_{y}=\mathcal{D}_{y} \mathcal{D}_{x}=\frac{\tau^{2}}{4 h_{x}^{\alpha} h_{y}^{\beta}}\left(K_{1}^{-} A_{\beta}+K_{2}^{-} A_{\beta}^{\mathrm{T}}\right) \otimes\left(K_{1}^{+} A_{\alpha}+K_{2}^{+} A_{\alpha}^{\mathrm{T}}\right) .
$$


Thus (4.18) can be rewritten as

$$
e^{n}=\left(\left(I-\mathcal{D}_{y}\right)^{-1}\left(I+\mathcal{D}_{y}\right)\right)^{n}\left(\left(I-\mathcal{D}_{x}\right)^{-1}\left(I+\mathcal{D}_{x}\right)\right)^{n} e^{0} .
$$

We can also calculate the symmetric parts of $\mathcal{D}_{x}$ and $\mathcal{D}_{y}$ by Lemma 4.3 as

$$
\begin{aligned}
& \frac{\mathcal{D}_{x}+\mathcal{D}_{x}^{\mathrm{T}}}{2}=\frac{\left(K_{1}^{+}+K_{2}^{+}\right) \tau}{2 h_{x}^{\alpha}} I_{y} \otimes\left(\frac{A_{\alpha}+A_{\alpha}^{\mathrm{T}}}{2}\right), \\
& \frac{\mathcal{D}_{y}+\mathcal{D}_{y}^{\mathrm{T}}}{2}=\frac{\left(K_{1}^{-}+K_{2}^{-}\right) \tau}{2 h_{y}^{\beta}}\left(\frac{A_{\beta}+A_{\beta}^{\mathrm{T}}}{2}\right) \otimes I_{x} .
\end{aligned}
$$

From Theorem 2.13 the eigenvalues of $\frac{A_{\alpha}+A_{\alpha}^{\mathrm{T}}}{2}$ and $\frac{A_{\beta}+A_{\beta}^{\mathrm{T}}}{2}$ are all negative when $1<\alpha, \beta \leq 2$. Defining $\lambda_{\alpha}$ and $\lambda_{\beta}$ as an eigenvalue of matrices $\mathcal{D}_{x}$ and $\mathcal{D}_{y}$, respectively, then it yields from the consequences of Lemma 2.10 and 4.1 that the real parts of $\lambda_{\alpha}$ and $\lambda_{\beta}$ are both less than zero. Since $\left(1+\lambda_{\alpha}\right) /\left(1-\lambda_{\alpha}\right)$ and $(1+$ $\left.\lambda_{\beta}\right) /\left(1-\lambda_{\beta}\right)$ are eigenvalues of matrices $\left(I-\mathcal{D}_{x}\right)^{-1}\left(I+\mathcal{D}_{x}\right)$ and $\left(I-\mathcal{D}_{y}\right)^{-1}\left(I+\mathcal{D}_{y}\right)$, respectively, thus the spectral radius of each matrix is less than 1 , which follows that $\left(\left(I-\mathcal{D}_{x}\right)^{-1}\left(I+\mathcal{D}_{x}\right)\right)^{n}$ and $\left(\left(I-\mathcal{D}_{y}\right)^{-1}\left(I+\mathcal{D}_{y}\right)\right)^{n}$ converge to zero matrix (see Theorem 1.5 in [21]). Therefore the difference scheme (4.6) is unconditionally stable.

Remark 4.5. For the similar reason described in Remark 3.2 and the proof of Theorem 4.4, we conclude that the WSGD scheme with $\theta$ weighted scheme for the time discretization for (4.1) is unconditionally stable when $\frac{1}{2} \leq \theta \leq 1$.

Lemma 4.6. Let $\mathcal{D}_{x}$ and $\mathcal{D}_{y}$ be defined in (4.17), then

$$
\begin{aligned}
& \left\|\left(I-\mathcal{D}_{x}\right)^{-1}\left(I-\mathcal{D}_{y}\right)^{-1}\right\|_{2} \leq 1, \\
& \left\|\left(I-\mathcal{D}_{\gamma}\right)^{-1}\left(I+\mathcal{D}_{\gamma}\right)\right\|_{2} \leq 1, \gamma=x, y,
\end{aligned}
$$

where $\|\cdot\|_{2}$ denotes the 2-norm (spectral norm).

Proof. From Theorem 2.13 and Lemma 4.1, we know that $\mathcal{D}_{x}+\mathcal{D}_{x}^{\mathrm{T}}$ and $\mathcal{D}_{y}+\mathcal{D}_{y}^{\mathrm{T}}$ are negative semi-definite and symmetric matrices. Then for any $v \in \mathbb{R}^{\left(N_{x}-1\right) \times\left(N_{y}-1\right)}$, we obtain that

$$
v^{\mathrm{T}} v \leq v^{\mathrm{T}}\left(I-\mathcal{D}_{\gamma}^{\mathrm{T}}\right)\left(I-\mathcal{D}_{\gamma}\right) v, \gamma=x, y .
$$

Substituting $v$ and $v^{\mathrm{T}}$ by $\left(I-\mathcal{D}_{\gamma}\right)^{-1} v$ and $v^{\mathrm{T}}\left(I-\mathcal{D}_{\gamma}^{\mathrm{T}}\right)^{-1}$, respectively, for any $v \in \mathbb{R}^{\left(N_{x}-1\right) \times\left(N_{y}-1\right)}$, we get

$$
v^{\mathrm{T}}\left(I-\mathcal{D}_{\gamma}^{\mathrm{T}}\right)^{-1}\left(I-\mathcal{D}_{\gamma}\right)^{-1} v \leq v^{\mathrm{T}} v, \gamma=x, y .
$$

Thus, it leads to

$$
\left\|\left(I-\mathcal{D}_{\gamma}\right)^{-1}\right\|_{2}=\sup _{v \neq 0} \sqrt{\frac{v^{\mathrm{T}}\left(I-\mathcal{D}_{\gamma}^{\mathrm{T}}\right)^{-1}\left(I-\mathcal{D}_{\gamma}\right)^{-1} v}{v^{\mathrm{T}} v}} \leq 1, \gamma=x, y .
$$

Consequently,

$$
\left\|\left(I-\mathcal{D}_{x}\right)^{-1}\left(I-\mathcal{D}_{y}\right)^{-1}\right\|_{2} \leq\left\|\left(I-\mathcal{D}_{x}\right)^{-1}\right\|_{2}\left\|\left(I-\mathcal{D}_{y}\right)^{-1}\right\|_{2} \leq 1
$$

holds.

Since $\mathcal{D}_{x}+\mathcal{D}_{x}^{\mathrm{T}}$ and $\mathcal{D}_{y}+\mathcal{D}_{y}^{\mathrm{T}}$ are negative semi-definite and symmetric, for any $v \in \mathbb{R}^{\left(N_{x}-1\right) \times\left(N_{y}-1\right)}$, we have

$$
v^{\mathrm{T}}\left(I+\mathcal{D}_{\gamma}^{\mathrm{T}}\right)\left(I+\mathcal{D}_{\gamma}\right) v \leq v^{\mathrm{T}}\left(I-\mathcal{D}_{\gamma}^{\mathrm{T}}\right)\left(I-\mathcal{D}_{\gamma}\right) v, \gamma=x, y
$$


By choosing vector $\left(I-\mathcal{D}_{\gamma}\right)^{-1} v$, we have that for any $v \in \mathbb{R}^{\left(N_{x}-1\right) \times\left(N_{y}-1\right)}$,

$$
v^{\mathrm{T}}\left(I-\mathcal{D}_{\gamma}^{\mathrm{T}}\right)^{-1}\left(I+\mathcal{D}_{\gamma}^{\mathrm{T}}\right)\left(I+\mathcal{D}_{\gamma}\right)\left(I-\mathcal{D}_{\gamma}\right)^{-1} v \leq v^{\mathrm{T}} v, \gamma=x, y .
$$

As $\left(I-\mathcal{D}_{\gamma}\right)^{-1}\left(I+\mathcal{D}_{\gamma}\right)=\left(I+\mathcal{D}_{\gamma}\right)\left(I-\mathcal{D}_{\gamma}\right)^{-1}$, then it yields that

$$
\begin{aligned}
\|(I- & \left.\mathcal{D}_{\gamma}\right)^{-1}\left(I+\mathcal{D}_{\gamma}\right)\left\|_{2}=\right\|\left(I+\mathcal{D}_{\gamma}\right)\left(I-\mathcal{D}_{\gamma}\right)^{-1} \|_{2} \\
& =\sup _{v \neq 0} \sqrt{\frac{v^{\mathrm{T}}\left(I-\mathcal{D}_{\gamma}^{\mathrm{T}}\right)^{-1}\left(I+\mathcal{D}_{\gamma}^{\mathrm{T}}\right)\left(I+\mathcal{D}_{\gamma}\right)\left(I-\mathcal{D}_{\gamma}\right)^{-1} v}{v^{\mathrm{T}} v}} \leq 1 .
\end{aligned}
$$

Theorem 4.7. Let $u_{i, j}^{n}$ be the exact solution of (4.1) with $1<\alpha, \beta \leq 2$, and $U_{i, j}^{n}$ the solution of the difference scheme (4.6), then for all $1 \leq n \leq M$, we have

$$
\left\|u^{n}-U^{n}\right\| \leq c\left(\tau^{2}+h_{x}^{2}+h_{y}^{2}\right)
$$

where $c$ denotes a positive constant and $\|\cdot\|$ stands for the discrete $L^{2}$-norm.

Proof. Let $e_{i, j}^{n}=u_{i, j}^{n}-U_{i, j}^{n}$, subtracting (4.5) from (4.6) leads to

$$
\left(I-\mathcal{D}_{x}\right)\left(I-\mathcal{D}_{y}\right) e^{n+1}=\left(I+\mathcal{D}_{x}\right)\left(I+\mathcal{D}_{y}\right) e^{n}+\tau \mathcal{E}^{n},
$$

where $\mathcal{D}_{x}$ and $\mathcal{D}_{y}$ are given in (4.17) and

$$
\begin{aligned}
e= & \left(e_{1,1}, e_{2,1}, \cdots, e_{N_{x}-1,1}, e_{1,2}, e_{2,2}, \cdots, e_{N_{x}-1,2}, \cdots,\right. \\
& \left.e_{1, N_{y}-1}, e_{2, N_{y}-1}, \cdots, e_{N_{x}-1, N_{y}-1}\right)^{\mathrm{T}}, \\
\mathcal{E}= & \left(\varepsilon_{1,1}, \varepsilon_{2,1}, \cdots, \varepsilon_{N_{x}-1,1}, \varepsilon_{1,2}, \varepsilon_{2,2}, \cdots, \varepsilon_{N_{x}-1,2}, \cdots,\right. \\
& \left.\varepsilon_{1, N_{y}-1}, \varepsilon_{2, N_{y}-1}, \cdots, \varepsilon_{N_{x}-1, N_{y}-1}\right)^{\mathrm{T}} .
\end{aligned}
$$

Since $\mathcal{D}_{x}$ commutes with $\mathcal{D}_{y}$, denoting $P=\left(I-\mathcal{D}_{x}\right)^{-1}\left(I-\mathcal{D}_{y}\right)^{-1}\left(I+\mathcal{D}_{x}\right)\left(I+\mathcal{D}_{y}\right)$, it yields that

$$
e^{n+1}=P e^{n}+\tau\left(I-\mathcal{D}_{x}\right)^{-1}\left(I-\mathcal{D}_{y}\right)^{-1} \mathcal{E}^{n} .
$$

Replacing $n$ by $k$ and iterating for all $0 \leq k \leq n-1$ and taking the discrete $L^{2}$-norm on both sides, we have that

$\left\|e^{n}\right\| \leq \tau\left\|\left(I-\mathcal{D}_{x}\right)^{-1}\left(I-\mathcal{D}_{y}\right)^{-1}\right\|_{2} \sum_{k=0}^{n-1}\left\|P^{k}\right\|_{2} \cdot\left\|\mathcal{E}^{n-1-k}\right\| \leq \tau \sum_{k=0}^{n-1}\left\|P^{k}\right\|_{2} \cdot\left\|\mathcal{E}^{n-1-k}\right\|$,

where Lemma 4.6 shows that $\left\|\left(I-\mathcal{D}_{x}\right)^{-1}\left(I-\mathcal{D}_{y}\right)^{-1}\right\|_{2} \leq 1$.

Since $\mathcal{D}_{x}$ and $\mathcal{D}_{y}$ commute, matrix $P$ can be rewritten as

$$
P=\left(I-\mathcal{D}_{x}\right)^{-1}\left(I+\mathcal{D}_{x}\right)\left(I-\mathcal{D}_{y}\right)^{-1}\left(I+\mathcal{D}_{y}\right) .
$$

We then obtain from Lemma 4.6 that

$$
\|P\|_{2} \leq\left\|\left(I-\mathcal{D}_{x}\right)^{-1}\left(I+\mathcal{D}_{x}\right)\right\|_{2}\left\|\left(I-\mathcal{D}_{y}\right)^{-1}\left(I+\mathcal{D}_{y}\right)\right\|_{2} \leq 1 .
$$

Then for any $1 \leq k \leq M,\left\|P^{k}\right\|_{2} \leq\|P\|_{2}^{k} \leq 1$ holds. Now we can get that

$$
\left\|e^{n}\right\| \leq \tau \sum_{k=0}^{n-1}\left\|\mathcal{E}^{k}\right\| \leq c\left(\tau^{2}+h_{x}^{2}+h_{y}^{2}\right)
$$

The convergence result for scheme (4.13) can also be obtained by the similar way as above. 


\section{NUMERICAL EXAMPLES}

\subsection{One-dimensional case.}

Example 5.1. Consider the following problem

$$
\frac{\partial u(x, t)}{\partial t}={ }_{0} D_{x}^{\alpha} u(x, t)-\mathrm{e}^{-t}\left(x^{1+\alpha}+\Gamma(2+\alpha) x\right), \quad(x, t) \in(0,1) \times(0,1],
$$

with the boundary conditions

$$
u(0, t)=0, \quad u(1, t)=\mathrm{e}^{-t}, \quad t \in[0,1],
$$

and initial value

$$
u(x, 0)=x^{1+\alpha}, \quad x \in[0,1] .
$$

Then the exact solution of (5.1) is $u(x, t)=\mathrm{e}^{-t} x^{1+\alpha}$.

In Table 2, we present the maximum and $L^{2}$ errors at $t=1$ and the corresponding convergence rates to Example 5.1 approximated by the CN-WSGD scheme (3.6) with different space step sizes. The second order accuracy in space is verified for the problem (3.1) with only left Riemann-Liouville fractional derivative, and the numerical results show that the scheme (3.6) also performs efficiently for $\alpha$ near 1 .

TABLE 2. The maximum and $L^{2}$ errors and their convergence rates to Example 5.1 approximated by the CN-WSGD scheme at $t=1$ for different $\alpha$ with $\tau=h$.

\begin{tabular}{rrcccccccc}
\hline & & \multicolumn{4}{c}{$(p, q)=(1,0)$} & \multicolumn{4}{c}{$(p, q)=(1,-1)$} \\
\cline { 3 - 10 }$\alpha$ & $N$ & $\left\|u^{M}-U^{M}\right\|_{\infty}$ & rate & $\left\|u^{M}-U^{M}\right\|$ & rate & $\left\|u^{M}-U^{M}\right\|_{\infty}$ & rate & $\left\|u^{M}-U^{M}\right\|$ & rate \\
\hline 1.1 & 16 & $6.65881 \mathrm{E}-05$ & - & $3.61993 \mathrm{E}-05$ & - & $9.07705 \mathrm{E}-04$ & - & $9.88412 \mathrm{E}-05$ & - \\
& 32 & $1.54190 \mathrm{E}-05$ & 2.11 & $8.91288 \mathrm{E}-06$ & 2.02 & $2.28231 \mathrm{E}-04$ & 1.99 & $1.69497 \mathrm{E}-05$ & 2.54 \\
& 64 & $3.59204 \mathrm{E}-06$ & 2.10 & $2.20864 \mathrm{E}-06$ & 2.01 & $5.54453 \mathrm{E}-05$ & 2.04 & $3.18905 \mathrm{E}-06$ & 2.41 \\
& 128 & $8.38779 \mathrm{E}-07$ & 2.10 & $5.50064 \mathrm{E}-07$ & 2.01 & $1.32272 \mathrm{E}-05$ & 2.07 & $6.62381 \mathrm{E}-07$ & 2.27 \\
& 256 & $2.07953 \mathrm{E}-07$ & 2.01 & $1.37309 \mathrm{E}-07$ & 2.00 & $3.12360 \mathrm{E}-06$ & 2.08 & $1.49541 \mathrm{E}-07$ & 2.15 \\
& 512 & $5.19919 \mathrm{E}-08$ & 2.00 & $3.43071 \mathrm{E}-08$ & 2.00 & $7.33195 \mathrm{E}-07$ & 2.09 & $3.55944 \mathrm{E}-08$ & 2.07 \\
\hline 1.5 & 16 & $6.17157 \mathrm{E}-05$ & - & $8.80121 \mathrm{E}-06$ & - & $3.88221 \mathrm{E}-04$ & - & $3.91200 \mathrm{E}-05$ & - \\
& 32 & $1.25568 \mathrm{E}-05$ & 2.30 & $2.30799 \mathrm{E}-06$ & 1.93 & $7.85748 \mathrm{E}-05$ & 2.30 & $5.04830 \mathrm{E}-06$ & 2.95 \\
& 64 & $2.47412 \mathrm{E}-06$ & 2.34 & $6.07043 \mathrm{E}-07$ & 1.93 & $1.54572 \mathrm{E}-05$ & 2.35 & $7.43659 \mathrm{E}-07$ & 2.76 \\
& 128 & $4.76404 \mathrm{E}-07$ & 2.38 & $1.56527 \mathrm{E}-07$ & 1.96 & $2.97507 \mathrm{E}-06$ & 2.38 & $1.49956 \mathrm{E}-07$ & 2.31 \\
& 256 & $9.01282 \mathrm{E}-08$ & 2.40 & $3.97926 \mathrm{E}-08$ & 1.98 & $5.62846 \mathrm{E}-07$ & 2.40 & $3.72282 \mathrm{E}-08$ & 2.01 \\
& 512 & $1.93161 \mathrm{E}-08$ & 2.22 & $1.00351 \mathrm{E}-08$ & 1.99 & $1.05033 \mathrm{E}-07$ & 2.42 & $9.60334 \mathrm{E}-09$ & 1.95 \\
\hline 1.9 & 16 & $1.63058 \mathrm{E}-05$ & - & $2.27814 \mathrm{E}-06$ & - & $6.02603 \mathrm{E}-05$ & - & $7.78084 \mathrm{E}-06$ & - \\
& 32 & $2.49190 \mathrm{E}-06$ & 2.71 & $6.49029 \mathrm{E}-07$ & 1.81 & $9.23273 \mathrm{E}-06$ & 2.71 & $9.04790 \mathrm{E}-07$ & 3.10 \\
& 64 & $4.93027 \mathrm{E}-07$ & 2.34 & $1.81207 \mathrm{E}-07$ & 1.84 & $1.35841 \mathrm{E}-06$ & 2.76 & $1.49823 \mathrm{E}-07$ & 2.59 \\
& 128 & $1.27340 \mathrm{E}-07$ & 1.95 & $4.81095 \mathrm{E}-08$ & 1.91 & $1.94436 \mathrm{E}-07$ & 2.80 & $4.02142 \mathrm{E}-08$ & 1.90 \\
& 256 & $3.23580 \mathrm{E}-08$ & 1.98 & $1.24022 \mathrm{E}-08$ & 1.96 & $3.06615 \mathrm{E}-08$ & 2.66 & $1.12278 \mathrm{E}-08$ & 1.84 \\
& 512 & $8.15631 \mathrm{E}-09$ & 1.99 & $3.14892 \mathrm{E}-09$ & 1.98 & $7.93775 \mathrm{E}-09$ & 1.95 & $2.99226 \mathrm{E}-09$ & 1.91 \\
\hline
\end{tabular}

Example 5.2. Consider the problem

$$
\begin{aligned}
& \frac{\partial u(x, t)}{\partial t}={ }_{0} D_{x}^{\alpha} u(x, t)+{ }_{x} D_{1}^{\alpha} u(x, t)+f(x, t), \quad(x, t) \in(0,1) \times(0,1], \\
& u(0, t)=u(1, t)=0, \quad t \in[0,1], \\
& u(x, 0)=x^{3}(1-x)^{3}, \quad x \in[0,1],
\end{aligned}
$$


with the source term

$$
\begin{aligned}
f(x, t)=-\mathrm{e}^{-t}\left(x^{3}(1-x)^{3}\right. & +\frac{\Gamma(4)}{\Gamma(4-\alpha)}\left(x^{3-\alpha}+(1-x)^{3-\alpha}\right) \\
& -3 \frac{\Gamma(5)}{\Gamma(5-\alpha)}\left(x^{4-\alpha}+(1-x)^{4-\alpha}\right) \\
& +3 \frac{\Gamma(6)}{\Gamma(6-\alpha)}\left(x^{5-\alpha}+(1-x)^{5-\alpha}\right) \\
& \left.-\frac{\Gamma(7)}{\Gamma(7-\alpha)}\left(x^{6-\alpha}+(1-x)^{6-\alpha}\right)\right) .
\end{aligned}
$$

By simple evaluation, the exact solution of (5.2) is $u(x, t)=\mathrm{e}^{-t} x^{3}(1-x)^{3}$.

Table 3 shows the maximum and $L^{2}$ errors at $t=1$ and the corresponding convergence rates to Example [5.2, obtained by the CN-WSGD scheme (3.6) with different space step sizes. We can observe that CN-WSGD scheme (3.6) with the two cases $(p, q)=(1,0)$ and $(1,-1)$ is also very efficient for the problem with two sided Riemann-Liouville fractional derivatives, and second order accuracy in space is achieved.

TABLE 3. The maximum and $L^{2}$ errors and their convergence rates to Example 5.2 approximated by the CN-WSGD scheme at $t=1$ for different $\alpha$ with $\tau=h$.

\begin{tabular}{rrcccccccc}
\hline & & \multicolumn{4}{c}{$(p, q)=(1,0)$} & \multicolumn{5}{c}{$(p, q)=(1,-1)$} \\
\cline { 3 - 10 }$\alpha$ & $N$ & $\left\|u^{M}-U^{M}\right\|_{\infty}$ & rate & $\left\|u^{M}-U^{M}\right\|$ & rate & $\left\|u^{M}-U^{M}\right\|_{\infty}$ & rate & $\left\|u^{M}-U^{M}\right\|$ & rate \\
\hline 1.1 & 16 & $1.21351 \mathrm{E}-04$ & - & $6.87244 \mathrm{E}-05$ & - & $1.04202 \mathrm{E}-04$ & - & $5.49761 \mathrm{E}-05$ & - \\
& 32 & $3.10400 \mathrm{E}-05$ & 1.97 & $1.75798 \mathrm{E}-05$ & 1.97 & $4.32767 \mathrm{E}-05$ & 1.27 & $2.00595 \mathrm{E}-05$ & 1.45 \\
& 64 & $7.93983 \mathrm{E}-06$ & 1.97 & $4.47207 \mathrm{E}-06$ & 1.97 & $1.48399 \mathrm{E}-05$ & 1.54 & $7.42486 \mathrm{E}-06$ & 1.43 \\
& 128 & $2.01674 \mathrm{E}-06$ & 1.98 & $1.12995 \mathrm{E}-06$ & 1.98 & $4.19788 \mathrm{E}-06$ & 1.82 & $2.23601 \mathrm{E}-06$ & 1.73 \\
& 256 & $5.08051 \mathrm{E}-07$ & 1.99 & $2.84150 \mathrm{E}-07$ & 1.99 & $1.10967 \mathrm{E}-06$ & 1.92 & $6.11319 \mathrm{E}-07$ & 1.87 \\
& 512 & $1.27511 \mathrm{E}-07$ & 1.99 & $7.12580 \mathrm{E}-08$ & 2.00 & $2.84899 \mathrm{E}-07$ & 1.96 & $1.59692 \mathrm{E}-07$ & 1.94 \\
\hline 1.5 & 16 & $2.03009 \mathrm{E}-04$ & - & $5.46438 \mathrm{E}-05$ & - & $2.99388 \mathrm{E}-04$ & - & $8.57787 \mathrm{E}-05$ & - \\
& 32 & $4.52559 \mathrm{E}-05$ & 2.17 & $1.37190 \mathrm{E}-05$ & 1.99 & $7.90624 \mathrm{E}-05$ & 1.92 & $2.31127 \mathrm{E}-05$ & 1.89 \\
& 64 & $1.13225 \mathrm{E}-05$ & 2.00 & $3.45401 \mathrm{E}-06$ & 1.99 & $2.01483 \mathrm{E}-05$ & 1.97 & $6.01008 \mathrm{E}-06$ & 1.94 \\
& 128 & $2.83579 \mathrm{E}-06$ & 2.00 & $8.67756 \mathrm{E}-07$ & 1.99 & $5.08147 \mathrm{E}-06$ & 1.99 & $1.53528 \mathrm{E}-06$ & 1.97 \\
& 256 & $7.09655 \mathrm{E}-07$ & 2.00 & $2.17555 \mathrm{E}-07$ & 2.00 & $1.27542 \mathrm{E}-06$ & 1.99 & $3.88274 \mathrm{E}-07$ & 1.98 \\
& 512 & $1.77509 \mathrm{E}-07$ & 2.00 & $5.44715 \mathrm{E}-08$ & 2.00 & $3.19447 \mathrm{E}-07$ & 2.00 & $9.76542 \mathrm{E}-08$ & 1.99 \\
\hline 1.9 & 16 & $2.02959 \mathrm{E}-04$ & - & $3.60448 \mathrm{E}-05$ & - & $2.35899 \mathrm{E}-04$ & - & $4.37067 \mathrm{E}-05$ & - \\
& 32 & $4.57927 \mathrm{E}-05$ & 2.15 & $8.97441 \mathrm{E}-06$ & 2.01 & $5.44882 \mathrm{E}-05$ & 2.11 & $1.10506 \mathrm{E}-05$ & 1.98 \\
& 64 & $9.36312 \mathrm{E}-06$ & 2.29 & $2.23928 \mathrm{E}-06$ & 2.00 & $1.13848 \mathrm{E}-05$ & 2.26 & $2.77301 \mathrm{E}-06$ & 1.99 \\
& 128 & $2.03859 \mathrm{E}-06$ & 2.20 & $5.59714 \mathrm{E}-07$ & 2.00 & $2.55286 \mathrm{E}-06$ & 2.16 & $6.94607 \mathrm{E}-07$ & 2.00 \\
& 256 & $5.08948 \mathrm{E}-07$ & 2.00 & $1.39944 \mathrm{E}-07$ & 2.00 & $6.35234 \mathrm{E}-07$ & 2.01 & $1.73827 \mathrm{E}-07$ & 2.00 \\
& 512 & $1.27160 \mathrm{E}-07$ & 2.00 & $3.49898 \mathrm{E}-08$ & 2.00 & $1.58420 \mathrm{E}-07$ & 2.00 & $4.34792 \mathrm{E}-08$ & 2.00 \\
\hline
\end{tabular}

Example 5.3. Consider the following variable coefficients problem:

$$
\begin{aligned}
\frac{\partial u(x, t)}{\partial t}= & x_{0}^{\alpha} D_{x}^{\alpha} u(x, t)+(1-x)^{\alpha}{ }_{x} D_{1}^{\alpha} u(x, t) \\
& +f(x, t), \quad(x, t) \in(0,1) \times(0,1], \\
u(0, t)= & u(1, t)=0, \quad t \in[0,1], \\
u(x, 0)= & x^{3}(1-x)^{3}, \quad x \in[0,1],
\end{aligned}
$$


with the source term

$$
\begin{array}{r}
f(x, t)=-\mathrm{e}^{-t}\left(x^{3}(1-x)^{3}+\frac{\Gamma(4)}{\Gamma(4-\alpha)}\left(x^{3}+(1-x)^{3}\right)-3 \frac{\Gamma(5)}{\Gamma(5-\alpha)}\left(x^{4}+(1-x)^{4}\right)\right. \\
\left.+3 \frac{\Gamma(6)}{\Gamma(6-\alpha)}\left(x^{5}+(1-x)^{5}\right)-\frac{\Gamma(7)}{\Gamma(7-\alpha)}\left(x^{6}+(1-x)^{6}\right)\right) .
\end{array}
$$

By simple evaluation, the exact solution of (5.3) is $u(x, t)=\mathrm{e}^{-t} x^{3}(1-x)^{3}$.

The numerical results for the variable coefficients problem in Example 5.3 are listed in Table 4 , including the maximum and $L^{2}$ errors at $t=1$ and the corresponding convergence rates. From Table 4 , second order accuracy in space is observed, which indicates the discretization formulae (2.16) with $(p, q)=(1,0)$ and $(1,-1)$ for the Riemann-Liouville fractional derivatives can also be efficient for designing finite difference scheme for some variable coefficients fractional problems.

TABLE 4. The maximum and $L^{2}$ errors and their convergence rates to Example 5.3 approximated by the CN-WSGD scheme at $t=1$ for different $\alpha$ with $\tau=h$.

\begin{tabular}{rrcccccccc}
\hline & & \multicolumn{4}{c}{$(p, q)=(1,0)$} & \multicolumn{4}{c}{$(p, q)=(1,-1)$} \\
\cline { 3 - 10 }$\alpha$ & $N$ & $\left\|u^{M}-U^{M}\right\|_{\infty}$ & rate & $\left\|u^{M}-U^{M}\right\|$ & rate & $\left\|u^{M}-U^{M}\right\|_{\infty}$ & rate & $\left\|u^{M}-U^{M}\right\|$ & rate \\
\hline 1.1 & 16 & $1.77123 \mathrm{E}-04$ & - & $7.32001 \mathrm{E}-05$ & - & $3.95613 \mathrm{E}-04$ & - & $1.92219 \mathrm{E}-04$ & - \\
& 32 & $4.47870 \mathrm{E}-05$ & 1.98 & $1.76184 \mathrm{E}-05$ & 2.05 & $9.75763 \mathrm{E}-05$ & 2.02 & $4.11452 \mathrm{E}-05$ & 2.22 \\
& 64 & $1.08962 \mathrm{E}-05$ & 2.04 & $4.36356 \mathrm{E}-06$ & 2.01 & $2.43654 \mathrm{E}-05$ & 2.00 & $1.00363 \mathrm{E}-05$ & 2.04 \\
& 128 & $2.66784 \mathrm{E}-06$ & 2.03 & $1.08906 \mathrm{E}-06$ & 2.00 & $6.10991 \mathrm{E}-06$ & 2.00 & $2.51523 \mathrm{E}-06$ & 2.00 \\
& 256 & $6.67126 \mathrm{E}-07$ & 2.00 & $2.72235 \mathrm{E}-07$ & 2.00 & $1.53026 \mathrm{E}-06$ & 2.00 & $6.31764 \mathrm{E}-07$ & 1.99 \\
\hline 1.5 & 16 & $1.88510 \mathrm{E}-04$ & - & $6.18902 \mathrm{E}-05$ & - & $3.56874 \mathrm{E}-04$ & - & $1.30433 \mathrm{E}-04$ & - \\
& 32 & $4.48741 \mathrm{E}-05$ & 2.07 & $1.46628 \mathrm{E}-05$ & 2.08 & $8.32954 \mathrm{E}-05$ & 2.10 & $2.80619 \mathrm{E}-05$ & 2.22 \\
& 64 & $1.10524 \mathrm{E}-05$ & 2.02 & $3.61334 \mathrm{E}-06$ & 2.02 & $2.02076 \mathrm{E}-05$ & 2.04 & $6.65178 \mathrm{E}-06$ & 2.08 \\
& 128 & $2.74933 \mathrm{E}-06$ & 2.01 & $8.99424 \mathrm{E}-07$ & 2.01 & $4.98975 \mathrm{E}-06$ & 2.02 & $1.63398 \mathrm{E}-06$ & 2.03 \\
& 256 & $6.86120 \mathrm{E}-07$ & 2.00 & $2.24518 \mathrm{E}-07$ & 2.00 & $1.24092 \mathrm{E}-06$ & 2.01 & $4.05976 \mathrm{E}-07$ & 2.01 \\
\hline 1.9 & 16 & $1.61881 \mathrm{E}-04$ & - & $4.02897 \mathrm{E}-05$ & - & $1.79407 \mathrm{E}-04$ & - & $5.75044 \mathrm{E}-05$ & - \\
& 32 & $3.43080 \mathrm{E}-05$ & 2.24 & $9.58213 \mathrm{E}-06$ & 2.07 & $4.06728 \mathrm{E}-05$ & 2.14 & $1.27751 \mathrm{E}-05$ & 2.17 \\
& 64 & $7.72475 \mathrm{E}-06$ & 2.15 & $2.35289 \mathrm{E}-06$ & 2.03 & $9.30708 \mathrm{E}-06$ & 2.13 & $3.02268 \mathrm{E}-06$ & 2.08 \\
& 128 & $1.91676 \mathrm{E}-06$ & 2.01 & $5.83977 \mathrm{E}-07$ & 2.01 & $2.34573 \mathrm{E}-06$ & 1.99 & $7.37420 \mathrm{E}-07$ & 2.04 \\
& 256 & $4.80573 \mathrm{E}-07$ & 2.00 & $1.45527 \mathrm{E}-07$ & 2.00 & $5.92611 \mathrm{E}-07$ & 1.98 & $1.82315 \mathrm{E}-07$ & 2.02 \\
\hline
\end{tabular}

\subsection{Two-dimensional case.}

Example 5.4. The following fractional diffusion problem

$$
\frac{\partial u(x, y, t)}{\partial t}={ }_{0} D_{x}^{1.2} u(x, y, t)+{ }_{x} D_{1}^{1.2} u(x, y, t)+{ }_{0} D_{y}^{1.8} u(x, y, t)+{ }_{y} D_{1}^{1.8} u(x, y, t)+f(x, y, t)
$$

is considered in the domain $\Omega=(0,1)^{2}$ and $t>0$ with boundary conditions $\left.u(x, y, t)\right|_{\partial \Omega}=0$ and the initial condition $u(x, y, 0)=x^{3}(1-x)^{3} y^{3}(1-y)^{3}$, where 
the source term

$$
\begin{aligned}
f(x, y, t)= & -\mathrm{e}^{-t}\left[\left(x^{3}(1-x)^{3} y^{3}(1-y)^{3}\right)\right. \\
& +\left(\frac{\Gamma(4)}{\Gamma(2.8)}\left(x^{1.8}+(1-x)^{1.8}\right)-\frac{3 \Gamma(5)}{\Gamma(3.8)}\left(x^{2.8}+(1-x)^{2.8}\right)\right. \\
& \left.+\frac{3 \Gamma(6)}{\Gamma(4.8)}\left(x^{3.8}+(1-x)^{3.8}\right)-\frac{\Gamma(7)}{\Gamma(5.8)}\left(x^{4.8}+(1-x)^{4.8}\right)\right) y^{3}(1-y)^{3} \\
& +\left(\frac{\Gamma(4)}{\Gamma(2.2)}\left(y^{1.2}+(1-y)^{1.2}\right)-\frac{3 \Gamma(5)}{\Gamma(3.2)}\left(y^{2.2}+(1-y)^{2.2}\right)\right. \\
& \left.\left.+\frac{3 \Gamma(6)}{\Gamma(4.2)}\left(y^{3.2}+(1-y)^{3.2}\right)-\frac{\Gamma(7)}{\Gamma(5.2)}\left(y^{4.2}+(1-y)^{4.2}\right)\right) x^{3}(1-x)^{3}\right] .
\end{aligned}
$$

Then the exact solution of the fractional partial differential equation is $u(x, y, t)=$ $\mathrm{e}^{-t} x^{3}(1-x)^{3} y^{3}(1-y)^{3}$.

We use four numerical schemes: LOD (4.14), PR-ADI (4.7), Douglas-ADI (4.8) and D'yakonov-ADI (4.9), to simulate Example 5.4, the maximum and $L^{2}$ errors and their convergence rates to Example 5.4 approximated at $t=1$ are listed in Table 5. where $N=N_{x}=N_{y}$, and $p, q$ are the shifted numbers of the WSGD operators. From the numerical results, three ADI schemes obtain more accurate

\begin{tabular}{|c|c|c|c|c|c|c|c|c|c|}
\hline \multirow[b]{2}{*}{ Scheme } & \multirow[b]{2}{*}{$N$} & \multicolumn{4}{|c|}{$(p, q)=(1,0)$} & \multicolumn{4}{|c|}{$(p, q)=(1,-1)$} \\
\hline & & $\left\|u^{M}-U^{M}\right\|_{\infty}$ & rate & $\left\|u^{M}-U^{M}\right\|$ & rate & $\left\|u^{M}-U^{M}\right\|_{\infty}$ & rate & $\left\|u^{M}-U^{M}\right\|$ & rate \\
\hline \multirow{5}{*}{ LOD } & 8 & $4.49810 \mathrm{E}-05$ & - & $1.36781 \mathrm{E}-05$ & - & $4.81859 \mathrm{E}-05$ & - & $1.50257 \mathrm{E}-05$ & - \\
\hline & 16 & $1.16951 \mathrm{E}-05$ & 1.94 & $3.68935 \mathrm{E}-06$ & 1.89 & $1.21720 \mathrm{E}-05$ & 1.99 & 3.77002E-06 & 1.99 \\
\hline & 32 & $2.94559 \mathrm{E}-06$ & 1.99 & $9.40245 \mathrm{E}-07$ & 1.97 & $3.11386 \mathrm{E}-06$ & 1.97 & $9.74178 \mathrm{E}-07$ & 1.95 \\
\hline & 64 & $7.36186 \mathrm{E}-07$ & 2.00 & $2.36472 \mathrm{E}-07$ & 1.99 & $7.84850 \mathrm{E}-07$ & 1.99 & $2.47973 \mathrm{E}-07$ & 1.97 \\
\hline & 128 & $1.83637 \mathrm{E}-07$ & 2.00 & $5.92494 \mathrm{E}-08$ & 2.00 & $1.96486 \mathrm{E}-07$ & 2.00 & $6.25130 \mathrm{E}-08$ & 1.99 \\
\hline \multirow{5}{*}{ PR-ADI } & 8 & $6.43195 \mathrm{E}-06$ & - & $1.95007 \mathrm{E}-06$ & - & $6.44770 \mathrm{E}-06$ & - & $2.05016 \mathrm{E}-06$ & - \\
\hline & 16 & $1.54712 \mathrm{E}-06$ & 2.06 & $4.84833 \mathrm{E}-07$ & 2.01 & $2.04790 \mathrm{E}-06$ & 1.65 & $6.06100 \mathrm{E}-07$ & 1.76 \\
\hline & 32 & $3.83522 \mathrm{E}-07$ & 2.01 & $1.21460 \mathrm{E}-07$ & 2.00 & $5.56723 \mathrm{E}-07$ & 1.88 & $1.69028 \mathrm{E}-07$ & 1.84 \\
\hline & 64 & $9.57751 \mathrm{E}-08$ & 2.00 & $3.04854 \mathrm{E}-08$ & 1.99 & $1.44070 \mathrm{E}-07$ & 1.95 & $4.50482 \mathrm{E}-08$ & 1.91 \\
\hline & 128 & $2.39462 \mathrm{E}-08$ & 2.00 & $7.64237 \mathrm{E}-09$ & 2.00 & $3.65748 \mathrm{E}-08$ & 1.98 & $1.16567 \mathrm{E}-08$ & 1.95 \\
\hline \multirow{5}{*}{$\begin{array}{l}\text { Douglas- } \\
\text { ADI }\end{array}$} & 8 & $6.43195 \mathrm{E}-06$ & - & $1.95007 \mathrm{E}-06$ & - & $6.44770 \mathrm{E}-06$ & - & $2.05016 \mathrm{E}-06$ & - \\
\hline & 16 & $1.54712 \mathrm{E}-06$ & 2.06 & $4.84833 \mathrm{E}-07$ & 2.01 & $2.04790 \mathrm{E}-06$ & 1.65 & $6.06100 \mathrm{E}-07$ & 1.76 \\
\hline & 32 & $3.83522 \mathrm{E}-07$ & 2.01 & $1.21460 \mathrm{E}-07$ & 2.00 & $5.56723 \mathrm{E}-07$ & 1.88 & $1.69028 \mathrm{E}-07$ & 1.84 \\
\hline & 64 & $9.57751 \mathrm{E}-08$ & 2.00 & $3.04854 \mathrm{E}-08$ & 1.99 & $1.44070 \mathrm{E}-07$ & 1.95 & $4.50482 \mathrm{E}-08$ & 1.91 \\
\hline & 128 & $2.39462 \mathrm{E}-08$ & 2.00 & $7.64237 \mathrm{E}-09$ & 2.00 & $3.65748 \mathrm{E}-08$ & 1.98 & $1.16567 \mathrm{E}-08$ & 1.95 \\
\hline \multirow{5}{*}{$\begin{array}{l}\text { D'yakonov- } \\
\text { ADI }\end{array}$} & 8 & $6.43195 \mathrm{E}-06$ & - & $1.95007 \mathrm{E}-06$ & - & $6.44770 \mathrm{E}-06$ & - & $2.05016 \mathrm{E}-06$ & - \\
\hline & 16 & $1.54712 \mathrm{E}-06$ & 2.06 & $4.84833 \mathrm{E}-07$ & 2.01 & $2.04790 \mathrm{E}-06$ & 1.65 & $6.06100 \mathrm{E}-07$ & 1.76 \\
\hline & 32 & $3.83522 \mathrm{E}-07$ & 2.01 & $1.21460 \mathrm{E}-07$ & 2.00 & $5.56723 \mathrm{E}-07$ & 1.88 & $1.69028 \mathrm{E}-07$ & 1.84 \\
\hline & 64 & $9.57751 \mathrm{E}-08$ & 2.00 & $3.04854 \mathrm{E}-08$ & 1.99 & 1.44070E-07 & 1.95 & $4.50482 \mathrm{E}-08$ & 1.91 \\
\hline & 128 & $2.39462 \mathrm{E}-08$ & 2.00 & 7.64237E-09 & 2.00 & $3.65748 \mathrm{E}-08$ & 1.98 & $1.16567 \mathrm{E}-08$ & 1.95 \\
\hline
\end{tabular}
solution than the LOD scheme, and it also reflects that the three ADI schemes are equivalent in two dimensional case.

TABLE 5. The maximum and $L^{2}$ errors and their convergence rates to Example 5.4 approximated at $t=1$ with $\tau=h_{x}=h_{y}$.

\section{Conclusion}

The paper provides the novel second order approximations for fractional derivatives, called the weighted and shifted Grünwald difference operator; it also suggests a direction to gain higher order discretization and compact schemes of fractional derivatives, e.g., 31. The discretizations are used to solve one- and two-dimensional 
space fractional diffusion equations; several numerical schemes are designed, their effectiveness are theoretically proved and numerically verified.

\section{ACKNOWLEDGEMENTS}

The authors thank Professor Yujiang Wu for his constant encouragement and support, and also the reviewer for his/her valuable comments and suggestions. This work was supported by the Program for New Century Excellent Talents in University under Grant No. NCET-09-0438, the National Natural Science Foundation of China under Grant No. 10801067 and No. 11271173, and the Fundamental Research Funds for the Central Universities under Grant No. lzujbky-2010-63 and No. lzujbky-2012-k26.

\section{REFERENCES}

[1] E. Barkai, CTRW pathways to the fractional diffusion equation, Chem. Phys. 284 (2002) $13-27$

[2] Cem Celik and Melda Duman, Crank-Nicolson method for the fractional diffusion equation with the Riesz fractional derivative, J. Comput. Phys. 231 (2012), no. 4, 1743-1750, DOI 10.1016/j.jcp.2011.11.008. MR2876585

[3] Raymond H. Chan, Toeplitz preconditioners for Toeplitz systems with nonnegative generating functions, IMA J. Numer. Anal. 11 (1991), no. 3, 333-345, DOI 10.1093/imanum/11.3.333. MR 1118960 (92f:65041)

[4] Raymond Hon-Fu Chan and Xiao-Qing Jin, An Introduction to Iterative Toeplitz Solvers, Fundamentals of Algorithms, vol. 5, Society for Industrial and Applied Mathematics (SIAM), Philadelphia, PA, 2007. MR2376196 (2008k:65001)

[5] A.V. Chechkin, R. Goreno, I.M. Sokolov, Retarding subdiffusion and accelerating superdiffusion governed by distributed-order fractional diffusion equations, Phys. Rev. E. 66 (2002) 046129

[6] Minghua Chen and Weihua Deng, A second-order numerical method for two-dimensional twosided space fractional convection diffusion equation, Appl. Math. Model. 38 (2014), no. 13, 3244-3259, DOI 10.1016/j.apm.2013.11.043. MR3207518

[7] Jim Douglas Jr. and Seongjai Kim, Improved accuracy for locally one-dimensional methods for parabolic equations, Math. Models Methods Appl. Sci. 11 (2001), no. 9, 1563-1579, DOI 10.1142/S0218202501001471. MR1872682 (2002i:65083)

[8] Vincent J. Ervin and John Paul Roop, Variational formulation for the stationary fractional advection dispersion equation, Numer. Methods Partial Differential Equations 22 (2006), no. 3, 558-576, DOI 10.1002/num.20112. MR2212226 (2006m:65265)

[9] Rudolf Gorenflo and Francesco Mainardi, Random walk models for space-fractional diffusion processes, Fract. Calc. Appl. Anal. 1 (1998), no. 2, 167-191. MR1656314 (99m:60117)

[10] Natalia Krepysheva, Liliana Di Pietro, and Marie-Christine Néel, Space-fractional advectiondiffusion and reflective boundary condition, Phys. Rev. E (3) 73 (2006), no. 2, 021104, 9, DOI 10.1103/PhysRevE.73.021104. MR2224791 (2007c:35086)

[11] Alan J. Laub, Matrix Analysis for Scientists \& Engineers, Society for Industrial and Applied Mathematics (SIAM), Philadelphia, PA, 2005. MR.2128817

[12] Mark M. Meerschaert and Charles Tadjeran, Finite difference approximations for fractional advection-dispersion flow equations, J. Comput. Appl. Math. 172 (2004), no. 1, 65-77, DOI 10.1016/j.cam.2004.01.033. MR.2091131 (2005h:65138)

[13] Mark M. Meerschaert and Charles Tadjeran, Finite difference approximations for two-sided space-fractional partial differential equations, Appl. Numer. Math. 56 (2006), no. 1, 80-90, DOI 10.1016/j.apnum.2005.02.008. MR2186432 (2006j:65244)

[14] Ralf Metzler and Joseph Klafter, The random walk's guide to anomalous diffusion: a fractional dynamics approach, Phys. Rep. 339 (2000), no. 1, 77, DOI 10.1016/S03701573(00)00070-3. MR 1809268(2001k:82082)

[15] Kenneth S. Miller and Bertram Ross, An Introduction to the Fractional Calculus and Fractional Differential Equations, A Wiley-Interscience Publication, John Wiley \& Sons Inc., New York, 1993. MR1219954(94e:26013) 
[16] D.C. Negrete, B.A. Carreras, V.E. Lynch, Front Dynamics in Reaction-Diffusion Systems with Levy Flights: A Fractional Diffusion Approach, Phys. Rev. Lett. 91 (2003) 018302

[17] Manuel Duarte Ortigueira, Riesz potential operators and inverses via fractional centred derivatives, Int. J. Math. Math. Sci., posted on 2006, Art. ID 48391, 12, DOI 10.1155/IJMMS/2006/48391. MR2251718 (2007f:31007)

[18] D. W. Peaceman and H. H. Rachford Jr., The numerical solution of parabolic and elliptic differential equations, J. Soc. Indust. Appl. Math. 3 (1955), 28-41. MR0071874 (17,196d)

[19] Igor Podlubny, Fractional Differential Equations, An Introduction to Fractional Derivatives, Fractional Differential Equations, to Methods of Their Solution and some of Their Applications, Mathematics in Science and Engineering, vol. 198, Academic Press Inc., San Diego, CA, 1999. MR:1658022 (99m:26009)

[20] Alfio Quarteroni and Alberto Valli, Numerical Approximation of Partial Differential Equations, Springer Series in Computational Mathematics, vol. 23, Springer-Verlag, Berlin, 1994. MR 1299729 (95i:65005)

[21] Alfio Quarteroni, Riccardo Sacco, and Fausto Saleri, Numerical Mathematics, 2nd ed., Texts in Applied Mathematics, vol. 37, Springer-Verlag, Berlin, 2007. MR2265914 (2007f:65001)

[22] Jinggang Qin and Tongke Wang, A compact locally one-dimensional finite difference method for nonhomogeneous parabolic differential equations, Int. J. Numer. Methods Biomed. Eng. 27 (2011), no. 1, 128-142, DOI 10.1002/cnm.1299. MR2791881(2012d:65171)

[23] W. R. Schneider and W. Wyss, Fractional diffusion and wave equations, J. Math. Phys. 30 (1989), no. 1, 134-144, DOI 10.1063/1.528578. MR974464(89m:45017)

[24] E. Sousaa, C. Li, A weighted finite difference method for the fractional diffusion equation based on the Riemann-Liouville derivative, (2011), arXiv:1109.2345 [math.NA]

[25] Z. Z. Sun, Numerical Methods of Partial Differential Equations (in Chinese), Science Press, Beijing (2005)

[26] Charles Tadjeran, Mark M. Meerschaert, and Hans-Peter Scheffler, A second-order accurate numerical approximation for the fractional diffusion equation, J. Comput. Phys. 213 (2006), no. 1, 205-213, DOI 10.1016/j.jcp.2005.08.008. MR2203439(2006j:65253)

[27] Charles Tadjeran and Mark M. Meerschaert, A second-order accurate numerical method for the two-dimensional fractional diffusion equation, J. Comput. Phys. 220 (2007), no. 2, 813823, DOI 10.1016/j.jcp.2006.05.030. MR2284325 (2008a:65159)

[28] Q. Yang, F. Liu, and I. Turner, Numerical methods for fractional partial differential equations with Riesz space fractional derivatives, Appl. Math. Model. 34 (2010), no. 1, 200-218, DOI 10.1016/j.apm.2009.04.006. MR2566688 (2010j:65206)

[29] Cai Hua Wang and Tong Ke Wang, Extended locally one-dimensional finite difference and finite element schemes for nonhomogeneous parabolic differential equations with nonhomogeneous boundary conditions (Chinese, with English summary), Numer. Math. J. Chinese Univ. 28 (2006), no. 2, 138-150. MR2295134(2008e:65269)

[30] Fuzhen Zhang, Matrix Theory, Basic Results and Techniques, 2nd ed., Universitext, Springer, New York, 2011. MR 2857760 (2012h:15001)

[31] Han Zhou, WenYi Tian, and Weihua Deng, Quasi-compact finite difference schemes for space fractional diffusion equations, J. Sci. Comput. 56 (2013), no. 1, 45-66, DOI 10.1007/s10915012-9661-0. MR.3049942

School of Mathematics and Statistics, Lanzhou University, Lanzhou 730000, People's Republic of China

E-mail address: twymath@gmail.com

School of Mathematics and Statistics, Lanzhou University, Lanzhou 730000, People's Republic of ChinA

E-mail address: zhouh2010@lzu.edu.cn

School of Mathematics and Statistics, Lanzhou University, Lanzhou 730000, People's Republic OF China

Current address: Department of Mathematics, Hong Kong Baptist University, Hong Kong,

P.R. China - and - Department of Mathematics, Utrecht University, Utrecht, the Netherlands

E-mail address: dengwh@lzu.edu.cn 